\title{
Electronic structure of
}

\section{metal phthalocyanines}

\section{on Ag (100)}

\section{Cornelius Krull}

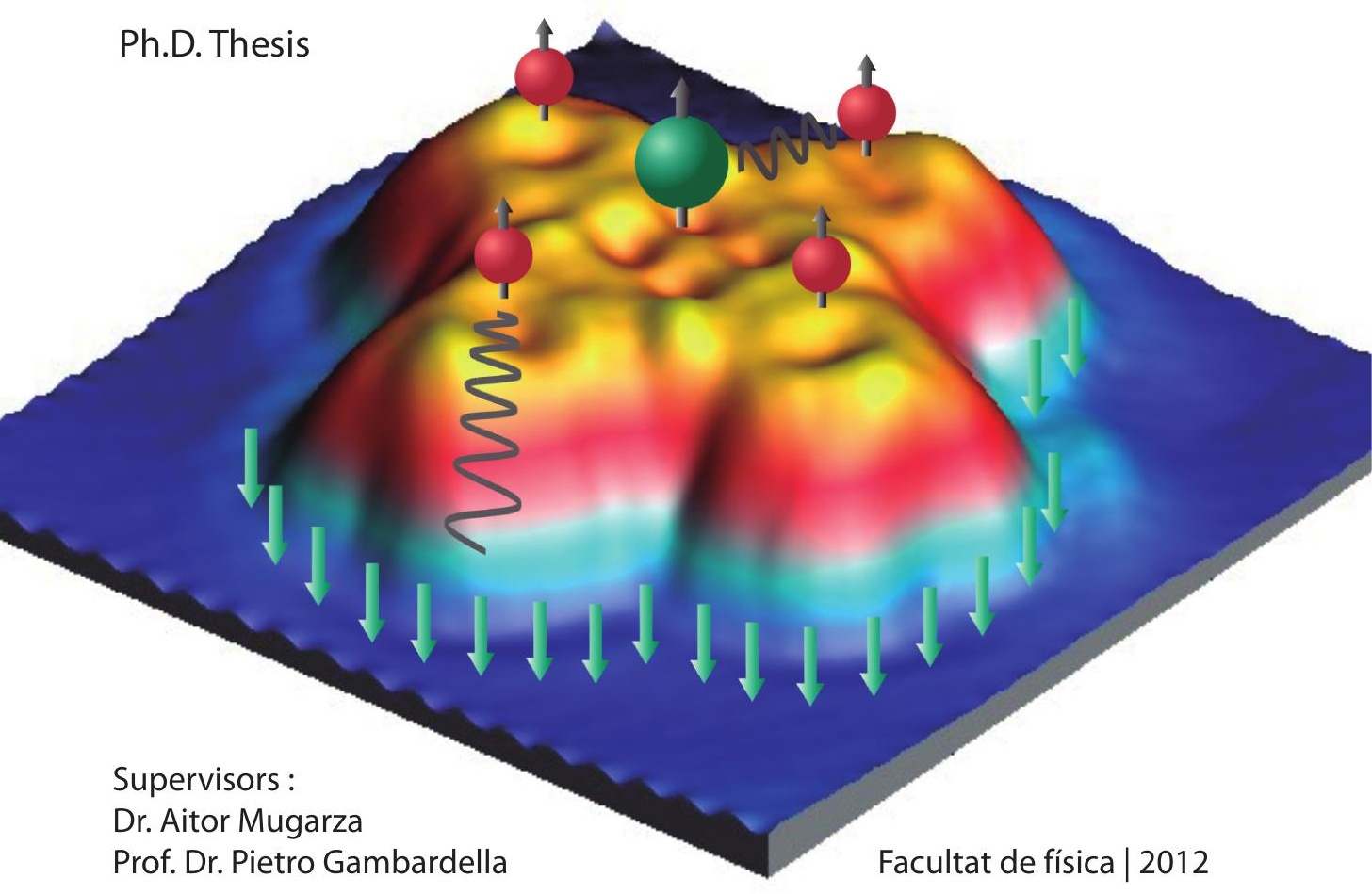

Institut Català de Nanotecnologia

Universitat Autònoma de Barcelona 
Cover images shows a 3D Scanning Tunneling Microscope topography image of Copper phthalocyanine adsorbed on a Silver (100) surface. Magnetic moments in the organic ligand and the ion center of the molecule are shown to couple to each other. The surface conduction electrons screen these moments through the Kondo interaction.

This document was typeset with LYX, the document processor. 


\section{A dissertation submitted to attain the degree of Doctor of Philosophy}

\section{Electronic structure of metal phthalocyanines on Ag (100)}

Cornelius Krull

2012

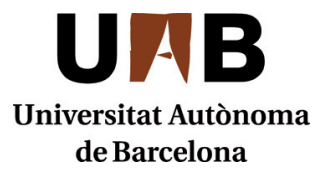

Facultat de Ciències - Física Institut Català de Nanotecnologia 



\section{Contents}

$\begin{array}{ll}\text { Abstract } & 1\end{array}$

$\begin{array}{ll}\text { Resumen } & 4\end{array}$

1. Introduction - Molecular electronics 7

2. Experimental methods 11

2.1. Scanning tunneling microscopy . . . . . . . . . . . 11

2.1.1. The operating principle . . . . . . . . . . 12

2.1.2. Theoretical descriptions of the tunneling process . . . 13

2.2. Scanning tunneling spectroscopy . . . . . . . . . . . 19

2.2.1. Elastic tunneling spectroscopy . . . . . . . . . . . . . . 19

2.2.2. Inelastic tunneling spectroscopy . . . . . . . . . . 20

2.2.3. Lock-in technique . . . . . . . . . . . . . . 22

2.2.4. Background subtraction _. . . . . . . . . . 23

2.2.5. Differential conductance maps . . . . . . . . . . . 25

2.3. Manipulation techniques . . . . . . . . . . . . 25

2.4. The experimental setup . . . . . . . . . . . . 28

2.5. Methods ... . . . . . . . . . . . . . 31

2.5.1. Sample preparation . . . . . . . . . . . 31

2.5.2. Density functional theory methods . . . . . . . . 32

3. Introduction to the Kondo effect 33

3.1. The Kondo problem . . . . . . . . . . . . . . . . . . . . 34

3.2. The Anderson model . . . . . . . . . . . . . . . . . 36

3.3. Virtual spin flips and the Schrieffer - Wolff transformation . 40

3.4. Formation of the Kondo singlet . . . . . . . . . . . . 42

3.4.1. The spin $\frac{1}{2}$ Kondo effect . . . . . . . . . . . . . . . 43

3.4.2. The underscreened Kondo effect . . . . . . . . . . . 47

3.4.3. The inelastic Kondo effect . . . . . . . . . . . . . . 49

3.5. The Kondo effect in STM measurements . . . . . . . . . . 51 
4. Adsorption of Metal Phthalocyanines on Ag(100) 55

4.1. Chemical structure of Metal Phthalocyanines . . . . . . . 56

4.2. Adsorption of single molecules . . . . . . . . . . . . 57

4.2.1. Adsorption configuration . . . . . . . . . . . . 57

4.2.2. Orbital specific electronic chirality . . . . . . . . 59

4.3. Monolayer growth . . . . . . . . . . . . . 63

4.3.1. Evolution of chirality in $\mathrm{CuPc}$ and CoPc structures . . 63

4.3.2. Supramolecular structure . . . . . . . . . . 66

4.3.3. Origin of the transfer of chirality . . . . . . . . 70

4.4. Multilayer growth . . . . . . . . . . . . 74

4.5. Summary . . . . . . . . . . . . . . . 76

5. Electronic and magnetic properties of MePc on Ag(100) 79

5.1. Pristine $\mathrm{MePc}$ - gas phase electronic structure . . . . . . . 81

5.2. Single molecules: electronic structure _. . . . . . . 83

5.2.1. Spectroscopy of molecular orbitals . . . . . . . . . 83

5.2.2. DFT: electronic structure . . . . . . . . . . . . 86

5.3. Single molecules: magnetic structure . . . . . . . . . 89

5.3.1. Kondo interaction . . . . . . . . . . . . . . . . 89

5.3.2. DFT: magnetic structure . . . . . . . . . . . . . 99

5.4. From clusters to monolayer . . . . . . . . . . . . . . 104

5.4.1. Small clusters of $\mathrm{CuPc}$. . . . . . . . . . . . . 104

5.4.2. Monolayers of $\mathrm{CuPc}$ and $\mathrm{CoPc} \ldots \ldots . . . . .108$

5.5. CuPc multilayer . . . . . . . . . . . . . 112

5.5.1. Molecules on higher layers - Spectroscopy . . . . . . 112

5.6. Summary . . . . . . . . . . . . . . . . . . . 119

6. Doping of MePc: Alkali and Fe atoms 123

6.1. Electron doping of $\mathrm{MePc}$. . . . . . . . . . . . . . . 123

6.1.1. Single MePc doped with Lithium . . . . . . . . . . 124

6.1.2. Li doping of a monolayer of $\mathrm{CuPc}$. . . . . . . . . . 140

6.2. Doping with Fe atoms . . . . . . . . . . . . . . 144

6.3. Summary . . . . . . . . . . . . . . . . . 150

7. Conclusions and Outlook 153

A. CuPcs on Au(111) 159

B. Adsorption of molecules on surface 161

B.1. Chemisorption and Physisorption . . . . . . . . . 161 
B.2. Weak and strong chemisorption . . . . . . . . . . 162

B.3. The role of the $\mathrm{d}$-band: Atomic adsorption . . . . . . . 164

B.4. Molecular adsorption . . . . . . . . . . . . . 167

B.5. Van der Waals forces . . . . . . . . . . . . . . . . . . 168

$\begin{array}{ll}\text { Nomenclature } & 171\end{array}$

$\begin{array}{ll}\text { Publications } & 173\end{array}$

$\begin{array}{ll}\text { Bibliography } & 175\end{array}$

$\begin{array}{ll}\text { Acknowledgments } & 203\end{array}$ 



\section{Abstract}

Traditional semiconductor technology will reach a size limit within the next years. A possible solution is the use of organic molecules in technological applications, which offer a number of key advantages: their intrinsic small size $(\sim \mathrm{nm})$ and ability to self assemble into functional structures. Moreover the highly developed methods of molecular synthesis allow to tailor their electronic and magnetic properties. However, the implementation of organic devices depends crucially on the understanding of the interaction between molecules and metal electrodes as well as moleculemolecule interactions.

Transition-metal phthalocyanines $(\mathrm{MePc})$ are well-known metal organic complexes, with bulk semiconducting properties. Their versatile chemistry combined with a relatively simple and robust structure, makes them the ideal system to study the interaction of metal-organic complexes with metal surfaces. This thesis deals with the structural electronic and magnetic properties of MePc molecules adsorbed on a metallic substrate. By using Scanning Tunneling Microscopy (STM) and Scanning Tunneling Spectroscopy (STS), we studied the molecule-substrate and molecule-molecule interaction for four $\mathrm{MePc}(\mathrm{Me}$ $=\mathrm{Fe}, \mathrm{Co}, \mathrm{Ni}, \mathrm{Cu}$ ) starting from single molecules up to multilayer films on a $\operatorname{Ag}(100)$ surface.

We found that single molecules adsorb in a planar fashion with their molecular axis rotated by $\pm 30^{\circ}$ with respect to the surface [011] direction. This rotation is caused by the bond optimization between the MePc's aza-N and underlying $\mathrm{Ag}$ atoms. Pristine MePc are achiral, however the mismatch between surface and molecular symmetry axis induces the formation of chiral orbitals in the structurally undistorted molecules. For $\mathrm{NiPc}$ and $\mathrm{CuPc}$ a bias dependent electronic chirality is observed. This effect shows that chirality can be manifest exclusively at the electronic level due to asymmetric charge transfer between molecules and substrate.

At submonolayer coverages the molecules self-assemble into clusters with a $5 \times 5$ R $37^{\circ}$ superstructure, and well defined organizational chirality. The single molecule chirality is univocally transferred to the supramolecular organization of the clusters by means of attractive vdW interactions. The monolayer consists of large homochiral domains that are delimited by the surface terraces. The formation of homochiral domains starting from a racemic mixture of clusters is due to a spontaneous symmetry breaking, induced by Ostwald ripening, and the thermally induced switching of 
molecular chirality.

By using STS, we investigated the electronic structure of four MePc adsorbed on $\mathrm{Ag}(100)$. Our systematic approach allowed us to study the charge transfer and hybridization mechanisms of $\mathrm{MePc}$ as a function of increasing occupancy of the $3 d$ metal states. We found that all four $\mathrm{MePc}$ receive approximately one electron from the substrate. However, depending on the central metal ion, charge transfer from the substrate has different consequences. In FePc and $\mathrm{CoPc}$, it mixes metal and ligand molecular orbitals, and induces a charge reorganization within the complete molecule. Whereas in $\mathrm{NiPc}$ and $\mathrm{CuPc}$ one electron is transferred to a ligand $\pi$-orbitals, leaving the $d$ states unperturbed.

STS spectra for $\mathrm{CuPc}$ and NiPc recorded at different positions within the molecule provide evidence of an intense zero bias Kondo resonance $\left(T_{K}=29 K\right)$ corresponding to the orbital extension of the ligand $2 e_{g}$ orbital (gas phase LUMO). This is direct evidence of an additional magnetic moment caused by the transfer of one electron to the $\pi$ states. We further find that this Kondo resonance couples to internal vibrations of the molecule in both NiPc and CuPc. For CuPc the presence of a magnetic moment in the ion's d-states as well as the ligand $\pi$ spin leads to a triplet ground state, owning to intramolecular exchange coupling between $d$ and $\pi$ electrons. Inelastic electron tunneling induces excitations of the higherenergy singlet state. For FePc and CoPc on the other hand, neither Kondo resonances nor inelastic excitations are observed. DFT calculations point to a mixed-valence state for both molecules, caused by the different spatial distribution of the frontier orbitals compared to $\mathrm{CuPc}$ and NiPc. For both $\mathrm{FePc}$ and $\mathrm{CoPc}$ the interaction with the substrate tends to reduce the magnetic moment.

We were able to investigate the influence of intermolecular forces on the electronic and magnetic structure as a function of neighboring molecules, by manipulating individual molecules to form artificial clusters. $\mathrm{CuPc}$ shows a complex evolution of the electronic structure. For CoPc clusters no changes occur, due to the possibly stronger substrate coupling through the TM-ion, which is less affected by intermolecular interactions.

Multilayers films of $\mathrm{CuPc}$ grow in a planar adsorption geometry. Between the 1st and 2 nd layer there is a $45^{\circ}$ rotation of the molecules with central ion aligned. Higher level show a lateral shift towards a ion$\mathrm{N}$ alignment, and a gradually increasing tilt. The electronic structure exhibits a gradual decoupling, and evolution towards a semiconducting behavior, manifested in the opening of a gap around $E_{F}$, the coupling to 
molecular vibrations, and voltage dependent tunneling barriers.

We studied the possibilities to manipulate the spin and change state of $\mathrm{MePc}$ through atom-by-atom doping with alkali electron donors. For $\mathrm{CuPc}$ we found that ligand and metal states can be selectively doped by changing the alkali-molecule bonding configuration. This permits tailoring of the spin $(0,1 / 2,1)$ and charge state (up to 2 extra electrons), with a single alkali dopant. Controlled manipulation of individual Li atoms further allowed us to explore the sequential charging behavior with up to 5 added $\mathrm{Li}$ atom and the effect of alkali ions on the Coulomb repulsion of molecular orbitals. The doping of CuPc monolayer films shows that the electrostatic interaction between Li atoms of neighboring Li@CuPc complexes leads to a crossover of stable configurations depending on the Li dosage, favoring the transfer to the ion states.

Finally, we investigated the effect of magnetic dopants such as Fe atoms on NiPc and $\mathrm{CuPc}$ molecules. No direct evidence of a coupling between the magnetic moments of $\mathrm{Fe}$ and $\mathrm{Cu}$ was observed. However the Kondo temperature of the Fe atoms varies due to changes in their local environment modulated by the presence of MePc.

Overall, these results provide a comprehensive view of the interaction of $\mathrm{MePc}$ with metal substrates as well as the magnetic and transport properties of molecular cluster, monolayer and multilayers. 
En los próximos años la tecnología tradicional de los semiconductores alcanzará su tamaño límite. Una alternativa es el uso de moléculas orgánicas, las cuales ofrecen una serie de ventajas como su tamaño $(\sim \mathrm{nm})$ y su capacidad de auto ensamblaje dando lugar a la formación de estructuras funcionales. Por otra parte, los métodos de síntesis moleculares permiten adaptar sus propiedades electrónicas y magnéticas. Sin embargo, la implementación en dispositivos orgánicos depende fundamentalmente de la comprensión de la interacción entre las moléculas y electrodos de metal, así como las interacciones molécula-molécula.

Las metal-ftalocianinas (MePc) son complejos metal-orgánicos con propiedades semiconductoras. Su versatilidad química combinada con una estructura simple y robusta, las convierte en un sistema ideal para estudiar la interacción de complejos metal-orgánicos con superficies metálicas. Esta tesis estudia las propiedades estructurales, electrónicas y magnéticas de las MePc adsorbidas sobre un sustrato metálico. Mediante el uso de microscopía de efecto túnel (STM) y espectroscopia de efecto túnel de escaneo (STS) se ha estudiado la interacción molécula-sustrato y molécula-molécula de cuatro $\mathrm{MePc}(\mathrm{Me}=\mathrm{Fe}, \mathrm{Co}, \mathrm{Ni}$ y $\mathrm{Cu})$ empezando desde moléculas individuales hasta multicapas de MePc en superficies de Ag (100).

Se ha observado que la adsorción molecular de las MePc es paralela al plano del sustrato y es impulsada por la optimización del enlace entre el ligando con el sustrato de Ag, dando lugar a dos posibles orientaciones del eje molecular en la superficie. El desajuste de simetría entre la molécula y el sustrato provoca una hibridación asimétrica de diferentes orbitales moleculares. Como resultado, la molécula aquiral muestra un contraste quiral en la topografía de STM, quedando intacta su estructura química. Las fuerza laterales de Van der Waals transfieren unívocamente la quiralidad electrónica de las moléculas individuales a niveles supramoleculares. La maduración de Ostwald provoca una ruptura espontanea de la simetría de la mezcla racémica inicial de los clústeres, obteniendo como resultado capas moleculares mesoscópicas homoquirales.

Las propiedades electrónicas y magnéticas dependen de toda la molécula (ligando e ión central). Todas las moléculas reciben aproximadamente un electrón de la superficie, y en función del tipo de ión central y del carácter de sus d-orbitales frontera, este puede comportarse de manera diferente. Para los estados-d de baja energía o los 
que se encuentren en el plano molecular, como ocurre en $\mathrm{NiPc} / \mathrm{CuPc}$, son los orbitales de los ligandos orgánicos los que aceptan el electrón adicional conllevando a su hibridación. Esto hace que el electrón no apareado cree un momento magnético adicional en estas moléculas dejando intacto el estado magnético de los iones centrales. Para la CuPc, la interacción de canje intramolecular entre los electrones $\mathrm{d}$ y $\pi$ conduce a la creación de un nuevo estado triplete $(S=1)$ en el estado fundamental. En cambio, en las moléculas de FePc y CoPc, la interacción a través de los estados-d fuera del plano molecular, provoca una reorganización compleja de carga dando lugar a la formación de un sistema de valencia mixta. En ambos casos esta interacción conduce a una reducción del momento magnético de los iones.

Se ha estudiado la posibilidad de manipular el estado de espín de las $\mathrm{MePc}$ dopando átomo por átomo con donadores de electrones alcalinos. Se ha encontrado que en las moléculas de $\mathrm{CuPc}$ es posible dopar selectivamente tanto los estados del ligando como los del metal cambiando la colocación del alcalino. Esto permite controlar el estado de espín (0, $1 / 2,1)$ y de carga (hasta 2 electrones adicionales) con un único dopante alcalino. La manipulación controlada de los átomos individuales de $\mathrm{Li}$ nos ha permitido explorar el comportamiento secuencial de carga con un máximo de cinco átomos de Li añadidos y el efecto de estos en la repulsión de Coulomb de los orbitales moleculares. El dopaje en monocapas de $\mathrm{CuPc}$ muestra que la interacción electrostática entre los átomos de Li de los complejos Li @CuPc vecinos conduce a un cruce de configuraciones estables en función de la dosis de Li, favoreciendo la transferencia a los estados de iones.

Por último, se ha investigado el efecto de los dopantes magnéticos como los átomos de Fe en las moléculas de NiPc y CuPc. Se ha observado que no hay ninguna evidencia directa de un acoplamiento entre los momentos magnéticos de Fe y Cu. Sin embargo, la temperatura de Kondo de los átomos de Fe varía debido a cambios locales en su entorno modulados por la presencia del MePc.

En general, estos resultados proporcionan una visión completa de la interacción de las MePc con sustratos metálicos, así como de las propiedades magnéticas y de transporte en clústeres moleculares, monocapas y multicapas. 



\section{1 \\ Chapter 1 \\ Introduction - Molecular electronics}

"Small is Beautiful.", is a statement coming originally from the world of economics [1]. It nevertheless holds true, when it comes to technology. Small is beautiful, because small is fast and small is cheap. In the 1950s the miniaturization of technological appliances, especially electronics, started at a fast pace, creating big markets. Miniaturized sensors, micromechanical devices and integrated circuits were developed quickly. In microelectronics the number of functional units, i.e., transistors roughly doubled each year, a statement known as Moore's law [2]. It became a driving force for the new semiconductor industry, to create ever smaller functional units in devices. Also in science the focus shifted towards smaller systems. Richard P. Feynman suggested in 1959 in his groundbreaking talk "There is plenty of room at the bottom" [3] the idea of "manipulating and controlling things in a smaller scale"; to achieve control over matter at the atomic or molecular level to create ever smaller functional units. Traditionally, and very successfully, this creation of "small functional units" is based on technologies that make big structures smaller, a method called "topdown”. But once the scale changes from micrometer to nanometer an intrinsic size limit is met. An alternative method consists of assembling functional units out of smaller building blocks in a "bottom-up" fashion. The smallest functional units, present in living organisms, are molecules. In biological systems, molecules perform an incredible number of different tasks. In technological applications the use of molecules may offer key advantages over the structuring of inorganic matter [4]: 
Structural perfection. Below a certain temperature threshold, usually at room temperature or higher, molecules are structurally perfect and identical, because chemistry stabilizes their composition.

Small size. Molecules already are in the size scale between 1 and 100 $\mathrm{nm}$, making them good building blocks for the construction of functional nanostructures with accompanying advantages in cost, efficiency, and power dissipation.

Self-assembly and recognition. In nature, molecular interactions lead to the self-assembly of complex and functional superstructures e.g. the DNA molecule. This can be used to create nanoscale structures by self-assembly $[5,6,7,8]$. Molecular recognition leads to changes in electronic behavior, providing the basis for both switching and sensing applications [9].

Dynamical stereochemistry. Many molecules have multiple distinct stable geometric structures or isomers. Such geometric isomers can have distinct optical and electronic properties, which in turn can be used to change the transport properties at the single molecular level, creating a molecular switch, e.g., azobenzene, which can by switched by light [10] and current [11].

Synthetic tailorability. The chemistry of molecular synthesis is highly developed. By varying the composition and geometry, it is possible to tailor make structural, bonding, transport, and optical properties of molecules [12].

However, the experimental and theoretical challenges to create devices based on functional molecules are enormous. The possible use of molecules as single functional units, or as an ordered self assembled layer, depends on the understanding and control of two key aspects: The interaction between the molecules themselves and the interaction with metallic electrodes or supporting substrate. Interesting and useful molecular properties can be destroyed, changed or newly created by both of these interactions. It is thus of paramount interest to study single molecules at a large variety of interfaces as well as their interaction in clusters and layer structures.

For thick organic layers $(\sim 100 \mathrm{~nm})$ the intermolecular interactions within the organic films, like van der Waals forces, dipolar interactions, donor-acceptor recognition, $\pi$-stacking, and hydrogen bonds, as well as the film-substrate interactions have been studied widely [13]. This knowledge has led to the creation of a range of different applications: organic-light emitting diodes (OLEDs) [14, 15], already commercialized as AMOLED displays, photovoltaic cells for solar energy generation 
$[16,17,18]$, gas sensors [19], and thin-film transistors [20].

Molecules are also interesting as simple electronic components. Already in 1974 Aviram and Ratner proposed the fabrication of an electronic rectifier based on a single molecule with a donor, spacer and acceptor structure [21]. Since then a lot of effort has been put into the investigation of molecules as functional electronic units. The conduction properties of molecules can be accessed by break junction experiments. Here the molecules are placed between two electrodes created by the mechanical fracturing of a wire or electromigration. These measurements are complex, as small changes in the somewhat uncontrolled geometry of the electrodes can lead to large changes in the conduction measurements. Despite these difficulties, interesting results were achieved, such as the fabrication of a single molecule transistor, showing a Coulomb blockade and the Kondo effect [22]. Moreover the inclusion of the spin in addition to charge to store and transport information leads to the concept of molecular spintronic. A promising pathway to create molecules for this line of research is ligand chemistry. Metal-organic complexes can be specifically constructed to have specific magnetic properties, making them interesting for molecular spintronic applications. Their hybrid nature can be used to induce room temperature magnetism by combining local and itinerant spins [23]. The spin states of a molecule can be modified by applying a gate voltage [24] or even by mechanically deforming the molecule inside the break junction [25].

This thesis focused on metal phthalocyanines, a well known type of coordination complexes formed by a central metal ion and an organic macrocyclic ligand. The discovery of these molecules dates back to the turn of the 20th century, when the purely organic (Pc) was first observed as a strongly colored reaction byproduct. The introduction of metal ion in the center of the Pc-molecule happened by accident in 1928. In a Scottish dye factory, a reaction vessel cracked and exposed the Pc-reagents to the outer steel piping, leading to the creation of a green-blue material. This material was FePc, a phthalocyanine ligand with an Fe ion in the center. The possible use of FePc, and metal phthalocyanines in general, as dye pigments became immediately apparent, and industrial production of them started in 1935 [26]. Academic interest also spurred and by 1936 the structure, synthesis, and capability of phthalocyanines molecules to complex with a wide range of metal ions were described.

More than 70 different metal atoms have been found to coordinate 
with phthalocyanines, creating a vast range of different electronic and magnetic properties. It is exactly this variety, paired with a simple and robust structure that makes them interesting for technological applications. Semiconducting bulk MePc crystals have already been used in thin film for OLED [27, 28] and solar cell devices [29, 30]. For the same reasons, in fundamental research MePcs have become a model system to study the interaction of metal-organic complexes with metal surfaces. Their flat adsorption geometry facilitates the bonding of both the central TM ion and organic ligands to the substrate, whereas their capability to coordinate many different metal atoms allows for a systematic investigation metal ion-dependent investigation of the magnetic properties.

Here we present a study of the MePc model system on a metallic substrate $\mathrm{Ag}(100)$. Starting at the level of individual molecules, we investigate the impact of molecule-substrate and molecule-molecule interactions on electronic and magnetic structure, and the self-assembly of MePc. The detailed characterization of larger structures clusters, monolayers, and multilayers systems allows us to shed light on the complex interplay between these interactions. The use of four different $\mathrm{MePc}(\mathrm{Me}=\mathrm{Fe}, \mathrm{Co}$, $\mathrm{Ni}, \mathrm{Cu}$ ) types allows us determine the role of the central ion in these processes. In a last step we explore the possibility of manipulating of the spin and charge states by doping the molecules with alkali metal atoms and $\mathrm{Fe}$ atoms.

The scanning tunneling microscope (STM) is an ideal tool to a better understanding of physical and chemical processes at the interface. STM provides information about the structural, electronic, and to some extent, magnetic properties on a sub-molecular scale. Therefore, in contrast to break junction experiments the molecule-electrode bonding configuration can be determined. Additionally, the ability of STM to manipulate the investigated system on the nanoscale allows the study of intermolecular interactions through the fabrication of different molecular structures. 


\section{Chapter 2 \\ Experimental methods}

In this chapter the experimental method - Scanning Tunneling Microscopy - used over the course of this thesis, will be briefly introduced. First the main concepts and their theoretical background are reviewed. Then a quick overview of the main experimental techniques like scanning tunneling spectroscopy and manipulation will be given, followed by a short description of the actual setup, and the sample preparations used throughout this thesis.

\subsection{Scanning tunneling microscopy}

Scanning Tunneling Microscopy (STM) is an experimental technique in surface physics that allows direct imaging of conducting or semiconducting surfaces on the nanometer scale. By using a local probe, images are acquired in real space complementing other techniques like Low Energy Electron Diffraction (LEED) that work in reciprocal space. Additionally, and more importantly perhaps, the STM provides spatially resolved access to the electronic structure of the sample. Since its development in 1981 [31, 32, 33], STM has become a powerful and widely used tool in surface science [34]. Further improvements like low temperature setups allowed the investigation of systems of absorbates, where a high mobility at room temperature would have eluded scanning, e.g. single molecules or adatoms. Especially these kind of setups promoted the use of STM not only as an imaging tool but also as means to manipulate the sample in a very precise way, for instance creating nanosized structures by moving single 
atoms and molecules [35, 36] or inducing localized chemical reactions [37].

\subsubsection{The operating principle}

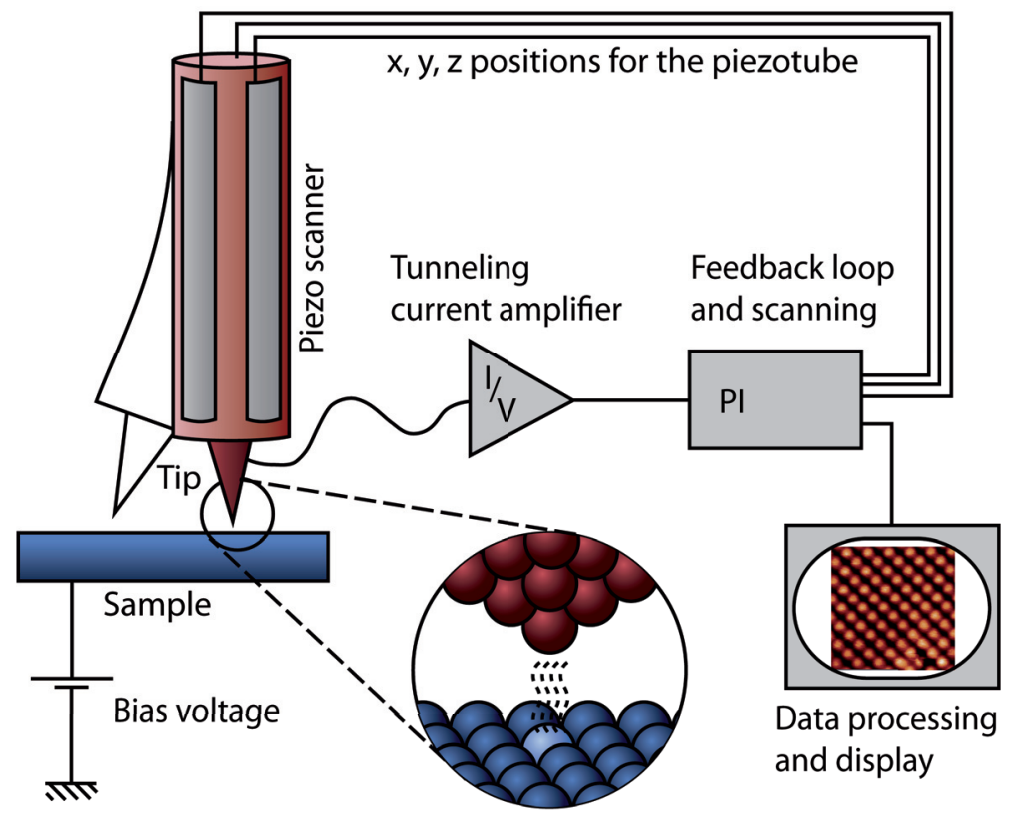

Figure 2.1.: The Scanning Tunneling Microscope: A sharp conducting tip is approached very close $(\sim 5 \AA)$ to the sample surface. Due to a bias voltage between tip an sample a current of tunneling electrons can be detected. Using a piezo electronic actuator the tip is moved in a scanning motion over the sample surface, resulting in a topographic image of the surface, based on the tunneling current signal.

The basic idea of an STM is based on the quantum mechanical tunnel effect. Consider a particle with a given energy that is confronted with a barrier in its trajectory. In classical mechanics it cannot cross the barrier if its potential is higher than its own energy. An example of this would be a ball trying to roll up a hill. However if one looks at a system that is small enough to require a quantum mechanical description the outcome of this experiment will be different. In quantum mechanics matter is seen as a wave and a particle at the same time. The position and the impulse 
of an object can only be determined with a certain degree of uncertainty. If the impulse is very sharply defined the position cannot be determined a vice versa; a concept known as the Heisenberg uncertainty principle. This implies that when a quantum mechanical particle, faces a potential energy barrier, the probability that the particle crosses the barrier cannot be zero, instead is decays exponentially over the width of the barrier. Hence there will always be a small probability for the particle to emerge on the other side of the barrier (see Figure 2.3). Naturally this probability will depend on the height of the barrier.

In an STM this effect is harnessed by approaching a very sharp metallic tip, ideally finished by just one atom, at a distance of about $0.1-1 \mathrm{~nm}$ to a conducting or at least semi-conducting sample. The gap between them is the barrier that electrons will tunnel though. In the absence of a bias, both directions for the tunneling from tip to the sample and vice versa are equally probable, and hence no net electron current would be detectable. But when a bias voltage between the sample and the tip is applied one tunneling direction is favored and a measurable current is detected. This tunneling current (typically $20 \mathrm{pA}$ to $10 \mathrm{nA}$ ) can be measured and depends exponentially on the distance between the sample and the tip.

Now if the tip scans an area on the surface at a constant height, the current signal is an image of the topography in real space. This is called the constant height scanning mode (see Figure 2.2). There are some disadvantages to this scanning mode. If the surface has topographic features higher than the scan height, the tip will crash against them changing the surface and the tip. Further the vertical height of the tip has to be very stable, with respect to thermal drift or coupling to external vibrations.

Another more commonly used scanning mode is the constant current mode (see Figure 2.2). In this case a feedback loop is used to maintain the current constant, by varying the height of the tip, i.e., the tunneling barrier. In this constant current mode, the currant channel will (ideally) be flat, and the topographic information is recorded in the height signal during the scan. Because the tip follows the profile of the surface this mode is better suited for a bigger corrugations. In this work almost exclusively the constant current mode was used.

\subsubsection{Theoretical descriptions of the tunneling process}

The tunneling process is described by a variety of different theoretical models. Two of which will be discussed in brief here. The first is a simple 


\section{Constant height}

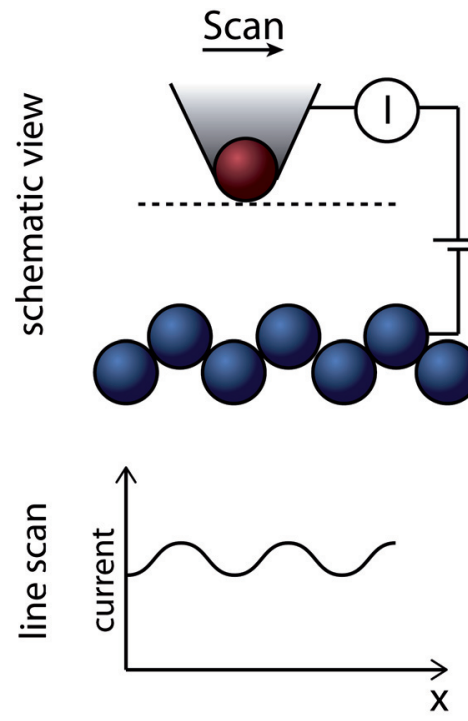

\section{Constant current}
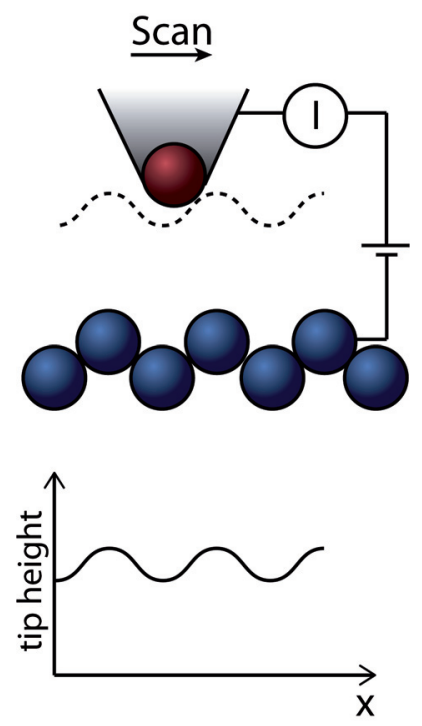

Figure 2.2.: The different STM scanning modes: In constant height mode tip is scanning at a constant height, the tunneling current signal contains the topographic data. In constant current mode the tunneling current is kept constant by a feedback loop controlling the tip height, the topographic signal is the tip height.

time-independent 1D model. Despite its approximative character this model is very instructive, because it can be solved analytically, and leads to the fundamental dependencies of the tunneling current. The second model takes all three dimensions into account, as well as the electronic structure of tip and sample.

\section{Simple 1D model}

In a first approach the tunnel junction can be modeled as a one dimensional and time independent system. The electron is approximated as a free electron with energy $E$, separated by a potential barrier of height $\Phi$ and thickness $d$.

The stationary Schrödinger equation (Equation 2.1) for the electron wave functions $\Psi$ in the tip, the sample and the barrier has to be solved. 


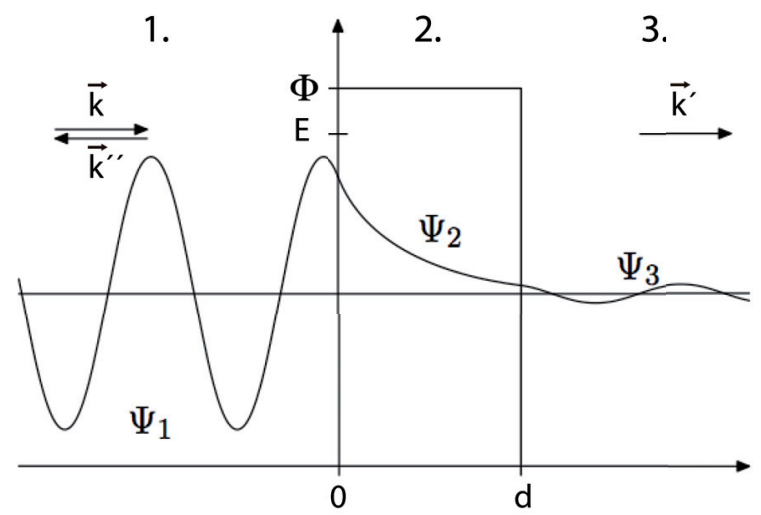

Figure 2.3.: One-dimensional potential barrier with energy $\Phi$ and width $d$. A particle that is traveling from the left to the right is described by three $\Psi$ before (1), inside (2), and after (3) the barrier. This leads to three wavenumbers $\vec{k}, \vec{k}^{\prime}$ and $\vec{k}^{\prime \prime}$. The $\vec{k}^{\prime}$ solution crossed the barrier.

$$
\left(\frac{\hbar^{2}}{2 m} \Delta+V(r)\right) \Psi=E \Psi
$$

The exact solution for these wave functions can be found by using a plane wave ansatz for $\Psi$ for of the three regions (tip (1), sample (3) and vacuum (2), see Figure 2.3):

$$
\begin{gathered}
\Psi_{1}=e^{i k z}+A \cdot e^{-i k z} \quad \Psi_{2}=B \cdot e^{i \kappa z}+C \cdot e^{-i \kappa z} \\
\Psi_{3}=D \cdot e^{i k z} \\
\text { with } k=\frac{\sqrt{2 m_{e} E}}{\hbar} \quad \text { and } \kappa^{2}=\frac{\sqrt{2 m_{e} \Phi-E}}{\hbar} \\
\text { where } m_{e} \text { is the electron mass }
\end{gathered}
$$

By matching the amplitude and the first derivative at the borders of the different potential regions, the coefficients (A, B, C, D) can be determined [38]. 
We can then define a transmission coefficient $T$, comparing the wavefunctions of both side of the barrier:

$$
T=\frac{\left|\Psi_{1}\right|^{2}}{\left|\Psi_{3}\right|^{2}}=\frac{A^{2}}{D^{2}}=\left[\left(\frac{k^{2}+\kappa^{2}}{2 k \kappa}\right)^{2} \sinh (\kappa d)\right]^{-1}
$$

which can be simplified for a high barrier potential compared to the energy of the electron $\Phi \gg E$, i.e., if $\kappa d \gg 1$.

$$
T \approx \frac{16 k^{2} \kappa^{2}}{\left(k^{2}+\kappa^{2}\right)^{2}} \cdot e^{-2 \kappa d}
$$

The number of tunneling electrons, the tunneling current $I$ will be proportional to $T$, therefore because the thickness of the barrier is given by the tip height $d$, we find:

$$
I \propto T \propto e^{-2 \kappa d}
$$

We have thus shown that the tunneling current depends exponentially on the tip sample distance $d$. The barrier height $\Phi$ be understood as the work function for both the sample and tip. For typical values for $\Phi=$ $5 \mathrm{eV}$ and $\mathrm{E}=2 \mathrm{eV}$, this means a change in the order of magnitude for $I$ for change of $1 \AA$ in $d$, in the tip sample distance. This exponential dependency is the underlying mechanism for the high vertical resolution of the STM technique.

\section{The Tersoff-Hamann model}

This model can be extended by including the electronic structure of the tip and sample and the three dimensions of the problem. Tersoff and Hamann $[39,40]$ presented this more realistic model in the 80 s. Their starting point was a first-order perturbation theory model introduced by Bardeen [41]. In this model the tunneling is described by $M_{\mu, \nu}$ the tunneling matrix i.e. the overlap of the wave functions of tip $\Psi_{\mu}$ and surface $\Psi_{\nu}$.

The tunneling current for a general geometry can then be written as:

$$
I=\frac{2 \pi e}{\hbar} \sum_{\mu, \nu} f\left(E_{\mu}\right)\left[1-f\left(E_{\nu}+e V\right)\right]\left|M_{\mu, \nu}\right|^{2} \delta\left(E_{\mu}-E_{\nu}\right)
$$




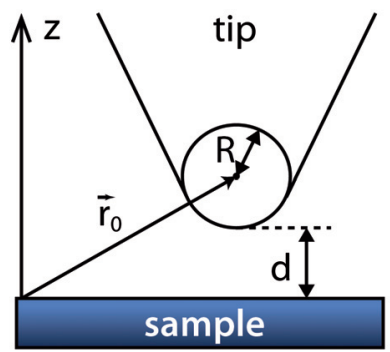

Figure 2.4.: Model used to approximate the wave function of the tip in the Tersoff Hamann description. The center of the tip is at $\vec{r}_{0}$ with an assumed spherical geometry, $R$ is the radius and $d$ the distance to the surface.

$$
f(E)=\frac{1}{1+\exp \left(\left(E-E_{F}\right) / k_{B} T\right)}
$$

Where $f(E)$ is the Fermi-Dirac distribution function, $M_{\mu, \nu}$ the tunneling matrix element and $E_{\mu}, E_{\nu}$ the energies of the states of surface and tip. $V$ is the applied bias voltage. In the limit of low temperatures and voltages $e \cdot V \ll \Phi$ Equation 2.6 can be simplified, because $f(E)$ becomes a step function with $f(E)=1$ for $E<E_{F}$ with $E_{F}$ the Fermi energy. We can thus approximate the $\left[1-f\left(E_{\nu}+e V\right)\right]$ term as a step function, this yields:

$$
I=\frac{2 \pi}{\hbar} e^{2} V \sum_{\mu, \nu}\left|M_{\mu, \nu}\right|^{2} \delta\left(E_{\mu}-E_{F}\right) \delta\left(E_{\nu}-E_{F}\right)
$$

The tunneling matrix element $\left(M_{\mu, \nu}\right)$, was shown by Bardeen [41] to be Equation 2.9 integrated over an arbitrary surface between tip and surface.

$$
M_{\mu, \nu}=-\frac{\hbar^{2}}{2 m} \int\left(\Psi_{\mu}^{*} \vec{\nabla} \Psi_{\nu}-\Psi_{\nu} \vec{\nabla} \Psi_{\mu}^{*}\right) d \vec{S}
$$

In order to arrive at quantitative tunneling currents it is necessary to define wave functions for surface and tip. These should describe the geometry found in an STM. The surface is described through a wave function parallel to the surface, consistent with Bloch's theorem. In the direction perpendicular it decays exponentially into the vacuum.

$$
\Psi_{\nu}=V_{\text {surface }}^{-1 / 2} \sum_{G} a_{G} \underbrace{e^{\left(-\sqrt{\kappa^{2}+\left|\vec{k}_{\|}+\vec{G}\right|^{2}} z\right)}}_{\text {Exp.decay }} \cdot \underbrace{e^{\left(i\left[\vec{k}_{\|}+\vec{G}\right] \vec{x}\right)}}_{\text {Blochwave }}
$$


Where $\vec{G}$ is a reciprocal lattice vector, $\kappa=\hbar^{-1}(2 m \Phi)$ the decay length into the vacuum with $\Phi$ the work function, and $\vec{k}_{\|}$the wave vector of the surface wave. $V_{\text {surface }}$ is the normalization volume of the surface. The tip is modeled as a spherical potential (see Figure 2.4) at the point closest to the surface, the rest is arbitrary. Accordingly the following wave function is used for the tip:

$$
\Psi_{\mu}=V_{\text {tip }}^{-1 / 2} \kappa R e^{\kappa R} \frac{1}{\kappa\left|\vec{r}-\vec{r}_{0}\right|} e^{-\kappa\left|\vec{r}-\vec{r}_{0}\right|}
$$

Again $V_{\text {tip }}$ is the normalization volume of the tip, $R$ the tip radius,$\kappa$ and $\Phi$ are the same constants as mentioned above. For simplicity's sake the work function of both tip and surface are assumed to be equal. With these model wave functions, Equation 2.10 and Equation 2.11, it is possible to simplify the tunneling matrix Equation 2.9 to:

$$
M_{\mu, \nu}=\frac{\hbar^{2}}{2 m} 4 \pi k^{-1} V_{\text {tip }}^{-1 / 2} k R e^{k R} \Psi_{\nu}\left(\vec{r}_{0}\right)
$$

Finally Equation 2.12 can be used to define the tunneling current Equation 2.8 and gives:

$$
I=32 \frac{\pi^{3}}{\hbar k^{4}} e^{2} V \Phi^{2} R^{2} e^{2 k R} \frac{1}{V_{\text {tip }}} \sum_{\mu \nu}\left|\Psi_{\nu}\left(\vec{r}_{0}\right)\right|^{2} \delta\left(E_{\mu}-E_{F}\right) \delta\left(E_{\nu}-E_{F}\right)
$$

To simplify this expression we note that the density of states for tip and surface are defined as follows:

$$
\begin{array}{r}
\rho_{\text {tip }}(E)=\frac{1}{V_{\text {tip }}} \sum_{\mu} \delta\left(E_{\mu}-E\right) \\
\rho_{\text {surface }}\left(E, \vec{r}_{0}\right)=\sum_{\nu}\left|\Psi_{\nu}\left(\vec{r}_{0}\right)\right|^{2} \delta\left(E_{\nu}-E\right)
\end{array}
$$

The final expression for the tunneling current comes to:

$$
I \propto V \rho_{\text {tip }}\left(E_{F}\right) \rho_{\text {surface }}\left(E_{F}, \vec{r}_{0}\right)
$$

Thus the current depends on the local density of states (LDOS) of the surface at the position of the tip $\vec{r}_{0}$ at the Fermi-Energy $E_{F}$. This means the STM images the LDOS of the surface rather then the positions of atoms. 
A more realistic description, a generalization of the Tersoff-Hamann model, defines the tunneling current by integrating over the states contributing to the tunneling current: the surface and tip DOS within the finite bias window. The dependence of the vacuum barrier on the tipsample distance $d$, the energy $E$ of each state, and the bias voltage $V$ is represented by a transmission coefficient $T(d, E, e V)[42,43,44]$.

$$
I \propto \int_{E_{F}}^{E_{F}+e V} \rho_{\text {surface }}\left(E_{F}-e V+\varepsilon\right) \rho_{\text {tip }}\left(E_{F}+\varepsilon\right) T(d, \varepsilon, e V) d \varepsilon
$$

We have thus found the contributing factors to the tunneling current: the DOS of tip and the sample and a transmission coefficient $T(d, E, e V)$. For small biases $\rho_{\text {tip }}$ and $T$ can be assumed constant; the tunneling current would then be primarily proportional to $\rho_{\text {surface }}$ integrated from $E_{F}$ to the applied bias voltage $E_{F}+V$.

However this model is still not a complete description of the tunneling process in an STM. Although in many standard situations e.g. in molecular adsorption studies it provides a reasonable qualitative picture. Some critical remarks have to be added nonetheless. The approximation of the tip as an $s$ orbital is rather inaccurate, as in tungsten tips the $d$ orbitals contribute to the major part of the tunneling current [45]. Also the interaction between tip and sample through microscopic chemical forces is not always negligible.

\subsection{Scanning tunneling spectroscopy}

\subsubsection{Elastic tunneling spectroscopy}

One of the key features of an STM setup is the ability to measure the electronic structure of a sample, locally at the position of the tip.

The basic principles of the scanning tunneling spectroscopy (STS) mode can be understood from Equation 2.17. If the transmission coefficient $T$ and to some extent $\rho_{\text {tip }}$ are assumed to be constant for the given energy interval, we can approximate the differential conductance $d I / d V$ can as:

$$
\frac{d I}{d V} \propto \rho_{\text {surface }}\left(E_{F}-e V\right) \cdot \rho_{\text {tip }}\left(E_{F}\right)
$$


The differential conductance $d I / d V$ is hence directly proportional to the local density of states of the sample at the position of the tip. Using this technique is thus possible to probe the electronic structure around $E_{F}$. Depending on the sign of applied bias the occupied or the unoccupied states are measured (See Figure 2.5).

In STM experiments the STS signal is obtained by positioning the tip over the point of interest, then the tunneling gap is set to appropriate current and voltage parameters, the feedback loop is opened to allow to the current to vary and the voltage is ramped to cover the region of interest. Typically the highest possible current setting that does not affect the sample is used. The voltage should be set to a region with relative constant DOS in all point investigated, to avoid strong variations in the tip height. This is, however, not always possible due to limitations in the measurement apparatus, and the risk of creating voltage pulses, if the change to the beginning of the voltage ramp is large. Hence usually, while not entirely correct the starting voltage of the voltage ramp was used in this work.

\subsubsection{Inelastic tunneling spectroscopy}

In addition to elastic tunneling through molecular states, electrons tunneling through molecules can couple to other internal degrees of freedom of the molecule. Examples of these are molecular vibrations [37], and spin excitations [46]. If an inelastic process can be excited, depends to various factors, the cross-section between molecular orbital and the process, the orientation of the molecule, the position of the tip etc. Once an electron has the required energy to excite the process, the interaction leads to the opening of another tunneling channel. Such processes lead to a change in the slope of an $I / V$ curve, hence a symmetric step function in the $d I / d V$ and peaks in the $d^{2} I / d V^{2}$ (Figure 2.5c). By recording the $d^{2} I / d V^{2}$ curve these excitations can be effectively measured. A combination of inelastic and elastic processes is also possible: Consider elastic tunneling through a molecular orbital, an electron with an energy larger than the resonance can excite vibronic modes. These modes are then detected as extra resonant peaks in the $d I / d V$ signal at energies multiple of the vibronic step above the molecular resonances (Figure 2.5b). 
a)

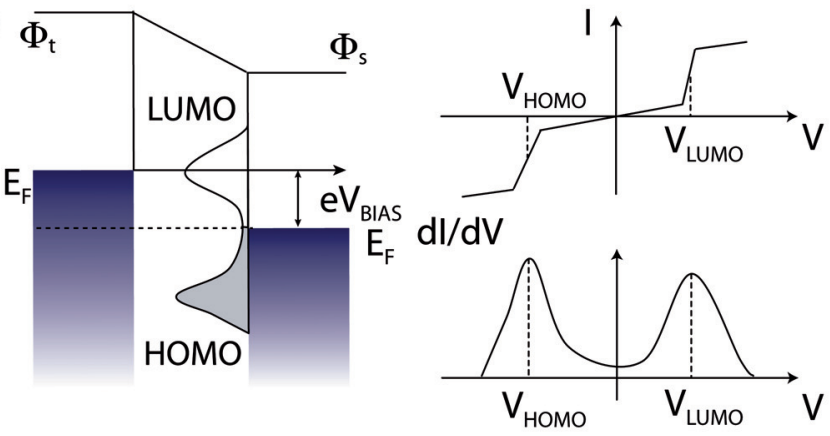

b)

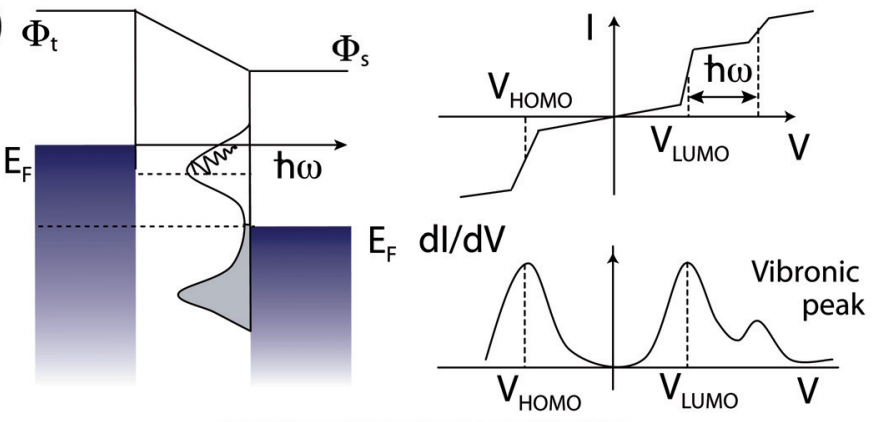

C)

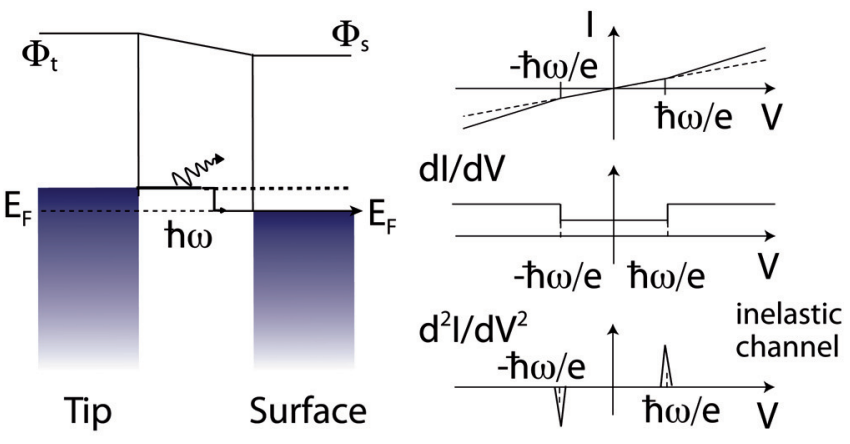

Figure 2.5.: STS tunneling through a molecule. a) Elastic tunneling at positive sample bias, electrons tunnel from the tip into the sample probing the unoccupied states around $E_{F}$ of the sample. The $d I / d V$ curve shows a peak at the position of the molecular orbitals. b) Tunneling process with vibronic excitation at a molecular orbital. The vibronic peak is represented as extra protrusions in the $d I / d V$ spectra at energy separations of the excited phonon. c) Inelastic tunneling. Electrons excite a vibration in the molecule and lose energy. The opening of this inelastic channel increases the conductance at the energy of the vibration. This is resolved as symmetric steps in the $d I / d V$ curve and peaks at opposite polarities in the $d^{2} I / d V^{2}$ spectrum. Energy level diagrams showing elastic and inelastic processes. $\Phi_{t}$ and $\Phi_{s}$ are the work functions of tip and sample. 


\subsubsection{Lock-in technique}

In an experimental setup the $d I / d V$ signal can be recorded directly using a lock-in amplifier. Compared to a numerical calculation from the $I / V$ curve, this method yields a better dynamic range and signal to noise ratio. In short the lock-in process is based on the orthogonality of sinusoidal functions. A small sinusoidal modulation $\left(V_{\text {mod }}=v_{o} \cos (\omega t)=1\right.$ to $100 \mathrm{mV}$ ) is applied to the bias voltage. Typically high frequencies which do not interfere with the topography measurements and are within the bandwidth of the current amplifier are used ( $f=1-3 \mathrm{kHz})$. The lock-in amplifier then multiplies the resulting measurement signal with a square wave of the exact same frequency using a phase shift, hereby effectively canceling contribution of other frequencies. This is done by integrating over short a period of time, the time constant $\tau(1 \mathrm{~ms}$ to $1 \mathrm{sec})$. The nature of the lock-in signal is such that the derivative of the signal is obtained. Higher derivatives can also be measured by varying the phase and using higher harmonics of the signal [47]. Thus for STS experiments the lock-in amplifier serves two proposes, reduction of the noise level and the direct derivation of the $I / V$ curve. The output of the lock-in is given by:

$$
V_{\text {out }}(t)=\int_{t-T_{c}}^{t} \sin \left(2 \pi f_{\text {ref }} \cdot s+\varphi\right) V_{\text {in }}(s) \cdot d s
$$

Two factors contribute to a broadening of the features in the DOS, $\tau$ and $V_{\text {mod. }}$. The value for $\tau$ has to be carefully chosen in relation to the time the voltage is ramped as this contributes to the intensity and energy resolution of the of the spectra. As a rule of thumb the resolution of an STS spectra is limited by the points of the spectra that were recorded for at least $5 \mathrm{x}$ $\tau$. For example a spectra taken over a range of $2000 \mathrm{mV}$ in 30 seconds, with $\tau=3 \mathrm{~ms}$, would have a resolution of $2000 \mathrm{mV} /(30 \mathrm{sec} / 5 \times 3 \mathrm{~ms})=1 \mathrm{mV}$ And secondly the amplitude of the modulation does have an effect on the maximum achievable spectral resolution as well. If one follows the math outlined in Refs. $[48,49]$ the broadening of spectral features is of the order of $\sqrt{2} \cdot V_{\text {mod }}$.

As we have seen in Equation 2.18 the STS signal is proportional to the density of states, therefore the intensities are usually measured in units of conductance [nanoSiemens]. The conversion between the lock-in output and conductance units follows directly,using the lock-in parameters like the full scale of the voltage output and the sensitivity . 


$$
\begin{gathered}
\frac{d I}{d V}=\left(\frac{\text { signal }}{\text { lock-in full scale }} \cdot \text { lock-in sensitivity } \cdot \text { preamplifier gain }\right) \\
\cdot \frac{1}{\text { lock-in modulation }}
\end{gathered}
$$

This means for typical units and gain settings that the signal will be in nanoSiemens:

$$
\frac{d I}{d V}=\frac{[\mathrm{V}]}{[\mathrm{V}]}[\mathrm{mV}][\mathrm{nA} / \mathrm{v}] /[\mathrm{mV}]=[\mathrm{nA} / \mathrm{v}]=[\mathrm{nS}]
$$

\subsubsection{Background subtraction}

Apart from the noise intrinsic to each particular STM setup due to vibrations, electronics and the broadening introduced by the lock-in, the major factor influencing line width and the shape of STS is the tip. The $d I / d V$ signal is convoluted with the DOS of the tip, which in real experiments is not constant. Therefore great care has to be taken to prepare the tip, not only to be sharp in order to achieve good topography resolution, but also for STS a tip with a featureless DOS is desirable. However the shape and DOS of the tip can only be manipulated in very indirect ways. A wide range of methods have been developed, e.g. by voltage pulses, or small controlled contacts with the surface, but all of them are not very reproducible and time intensive. The results will vary from tip to tip, and the tip may even change in between STS measurements.

A very efficient method to reduce the impact of tip DOS features is background subtraction. The basic idea is to measure the LDOS of the tip and the surface on a clean spot of the sample surface using the same feedback conditions as for the sample feature of interest. This data can then be used to deconvolute effects on the STS taken on the point of interest. Theoretically a mathematical deconvolution operation would be required to remove tip and surface contribution of the STS. However, as P.Wahl et al. [50] pointed out, a subtraction of the two STS curves is often sufficient, and leads to good results if the tip reference spectra does not present any sharp features. Throughout this work all background subtracted spectra are obtained in this manner. In Figure 2.6 this method is demonstrated, after subtraction the spectra taken with different tips are essentially the same. Furthermore the tip related feature appearing 

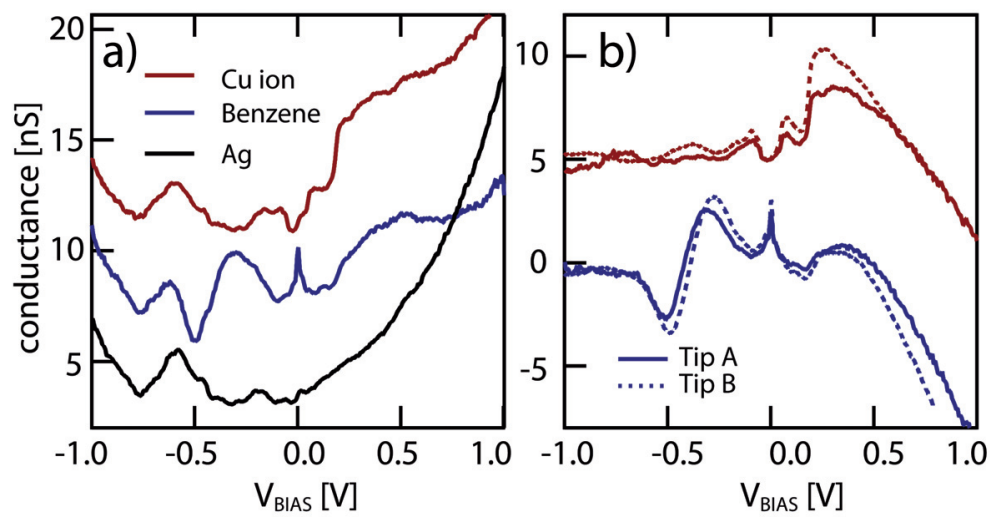

Figure 2.6.: a) $d I / d V$ spectra acquired on the $\mathrm{Cu}$ ion (red), and benzene (blue) of a single CuPc molecule, and on a clean spot of the Ag surface (black) with tip A (setpoint: $3.0 \mathrm{nA},-1.0 \mathrm{~V}$ ). The spectra have been y-offset for clarity, all of them start $\sim 10 \mathrm{nS}$, like the blue spectra. b) The same ion and benzene spectra after subtraction of the Ag spectrum (solid lines). For comparison, we show a pair of background subtracted spectra (dotted lines) acquired with a different tip (B) and initial setpoint ( $3.0 \mathrm{nA},-2.0 \mathrm{~V}$ ). The intensity of the latter have been reduced by a factor of 0.5 to account for the different initial setpoint. The spectra recorded on the Cu ion have been vertically shifted by $5 \mathrm{nS}$ for better visibility.

around $-0.6 \mathrm{~V}$ in the raw spectrum is removed, showing the efficacy of this method.

Nonetheless there certain are artifacts that can be created by this method, i.e., strong tip features can lead to artificial peaks in the resulting curves. Furthermore the conductance values reported for the spectra, represent relative values, negative values mean that the conductance at the point of interest at a certain energy is lower than that of the substrate (See Figure 2.6b). In conclusion it is not always feasible to apply this technique, depending on the investigated system, e.g., if the substrate is strongly modified by adsorbates, or if the tip has very pronounced features. 


\subsubsection{Differential conductance maps}

An intriguing application of an STM setup lies in the possibility to spatially resolve the topography of subnanoscale systems. The technique can be combined with STS, mapping the spatial distribution of features found in STS. The basic method is to scan an area of interest and take the $d I / d V$ intensity at every point of the scan. The resulting $d I / d V$ map is to some extent an image of the spatial distribution of the state investigated. The speed of such an acquisition is limited by the $\tau$ setting of the lock-in amplifier (5-8 $\tau$ per point) and the pixel resolution of the $d I / d V$ map. Depending on its size the measurement time varies between 5-30 min, meaning that the effect of thermal drift become more important. To counteract this usually the constant current mode is employed*, as well as lower resolution compared to topographic images is used.

The interpretation of such $d I / d V$ maps is however not straightforward, because the topography of the sample has a strong influence on the height of the tip and will enhance or reduce certain spectral features. A variety of normalization techniques for $d I / d V$ and $d I / d V$ maps exist, and provide useful results in certain particular situations. However none is these methods is generally applicable as can be seen by the large number of proposed methods $[44,51,52,53]$. Therefore in this work no normalization techniques were used. The qualitative comparison of molecular states for similar molecules in different environments, as presented in later chapters, is not severely be affected by topography effects.

\subsection{Manipulation techniques}

A powerful feature of an STM setup at low temperature is the possibility to locally manipulate the investigated system, by moving atoms or molecules with the tip. This is a great tool for scientific research as it allows control of the positions of single atoms and molecules to create new nanosystems and investigate them. The manipulation process is controlled through three main parameters in the STM: the electric field, the tunneling current, and the forces between tip and surface $[54,36]$. Care has to be taken to find for each specific system the right combination of parameters. There

\footnotetext{
*However with carefully chosen parameters a constant height map is also possible.
} 

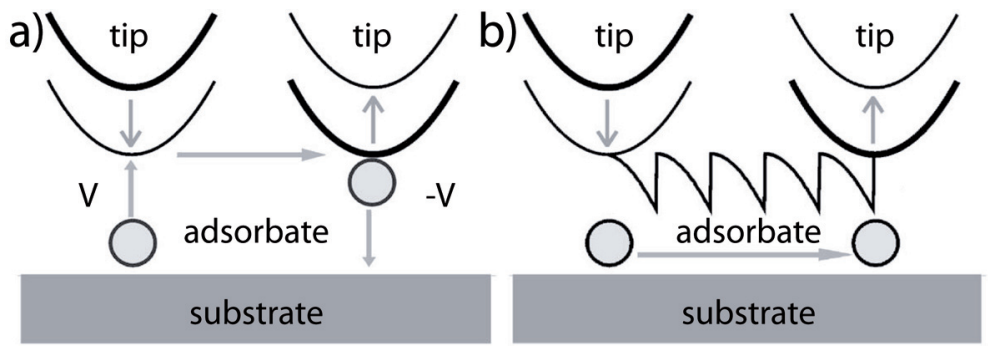

Figure 2.7.: a) Sketch of the vertical manipulation procedure: 1 . the tip is placed above the molecule/atom 2. to transfer an adsorbate to the tip a voltage pulse is applied while moving the tip towards the atom 3 . the tip is retracted and moved to the desired position 4 . the reverse pulse from the pick up is applied to drop the adsorbate. b) The lateral manipulation process in constant current mode: 1 . the tip is placed above the molecule/atom 2. the tip is approached towards the atom by increasing the current and decreasing the bias voltage 3. the tip is moved the desired pathway. The tip height curve shows the movement of the adsorbate on the surface. Images adapted from [36].

are two main methods employed for manipulation: vertical and lateral manipulation (see Figure 2.7).

In the vertical manipulation mode, field and current effects play a major role. Here the tip is also approached towards the adsorbate while a voltage pulse is applied. With the correct parameters this transfers the absorbate to the tip, which is effectively "picked up". Afterwards the tip is retracted and moved to a new location where a reverse pulse and approach drops the adsorbate again. In a related application this method can be used to modify the tip, to gain access to different spectral features due to changes of the symmetry of tunneling orbitals [55].

In the lateral manipulation mode, the tip is approached towards the surface, on top of the atom/molecule that has to be moved, by increasing the current and lowering the bias voltage (e.g. for $\mathrm{Li}$ on $\mathrm{Ag}(100) 50 \mathrm{mV}$ 250nA). Then the feedback is turned off and the tip follows the desired pathway and finally is retracted again. If the interaction between tip and atom, such as vdW, chemical forces or the electric field, is strong enough the atom is moved along with the tip. The molecules can either be pushed or pulled depending on the specific system, leading to characteristic tip height curves. A pulling movement, consists of a step increase as the molecule is pulled under the tip, and subsequent slow decrease as the tip 

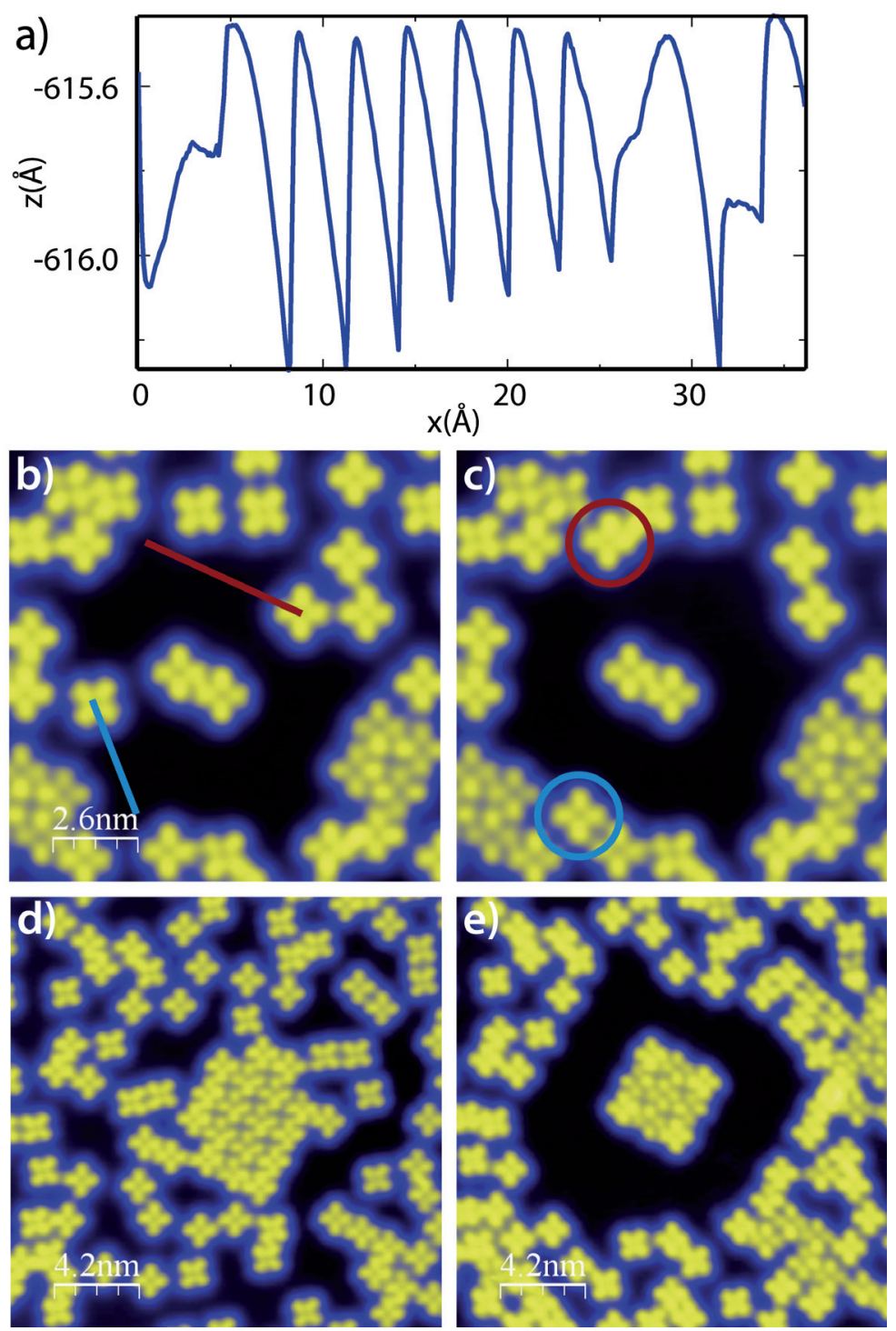

Figure 2.8.: a) Manipulation signals corresponding to a pulling movement of CuPc molecules adsorbed on $\mathrm{Ag}(100) \mathrm{b}$ ) and c) Images before and after dragging two CuPc molecules. A gap resistance of $333 \mathrm{~K} \Omega(150 \mu \mathrm{A}, 50 \mathrm{mV})$ was used for manipulation, and $1000 \mathrm{M} \Omega(0.1 \mathrm{nA}, 100 \mathrm{mV})$ for imaging. d) and e) Images before and after the formation of a $3 \times 3$ square cluster (see subsection 5.4.1). 
moves away from the it (See Figure 2.8a). For a pushing manipulation the curve would be mirrored, starting with a slow rise, followed by a sharp drop as the molecule jumps forward.

Both types of manipulation were used for the first time by the group of Don Eigler at IBM-Almaden [56] and later in Berlin [57] to build nanostructures from single atoms and molecules. Throughout this work mainly the lateral manipulation mode has been used to move atoms and molecules. In Figure 2.8 the creation of a $3 \times 3$ cluster of CuPc molecules is shown.

\subsection{The experimental setup}

The experimental setup used in this work is a low temperature STM based on a design by G.Meyer [58], and commercially available from Createc Fischer \& Co GmbH Berlin, Germany [59]. The system operates in an ultra high vacuum (UHV) environment ( $p<2 \cdot 10^{-10} \mathrm{mBar}$ ), and is based on liquid helium cryostat to work at temperatures $\sim 5 \mathrm{~K}$. In this section a brief review of the capabilities and limitations of this STM will be presented.

Figure 2.9 shows the vacuum chamber of the STM, it consists of two chambers, one used for sample preparation and a second that houses the STM and its cryostat. The UHV is generated by an array of pumps: two ion pumps one in each chamber, a turbo-molecular pump and a titan sublimation pump on the preparation chamber, and finally the cryostat which acts as cryo pump in the STM chamber.

\section{The cooling system}

The low operation temperatures are achieved by the use of a bath cryostat, made of two tanks. The inner one holds up to 11 liters of liquid helium while the outer is filled with liquid nitrogen. Both cooling stages have aluminum radiation shields that enclose the STM sample stage within. Using this method the sample can be kept cool and measured for up to $120 \mathrm{~h}$ per 1 refill of the helium deposit. The nitrogen tank lasts for approximately $48 \mathrm{~h}$.

\section{Scanner operation}

As introduced in section 2.1 on page on page 11 the basic idea of an STM is to place a sharp metallic tip very close to a surface, and then 

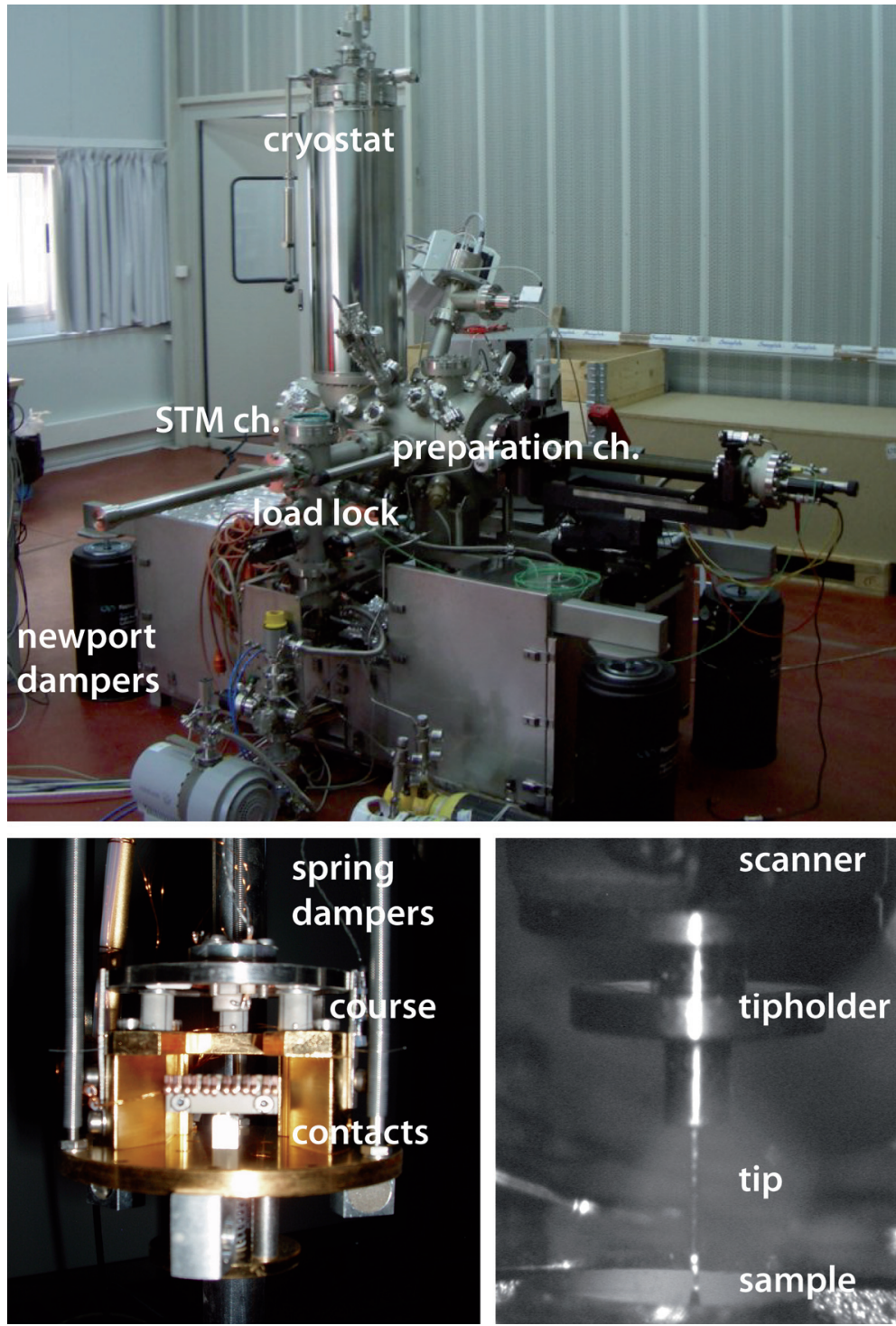

Figure 2.9.: a)The low temperature STM vacuum chamber: The STM is located below the cryostat, the preparation chamber features a sputter gun for cleaning the samples, a LEED optic, and several evaporators: The OMBE quadruple for molecular sources, a triple Omicron for metals. The STM chamber as an in-situ Alkali evap. and a single metal evap. b)The low temperature STM: the sample stage is shown: The sample holder is placed between the course motion ramp and the the contacts. the whole setup is hanging on spring to decouple from external vibrations. c) the piezo scanner, the scan piezo moves the tip in scanning moving over the surface. 
scan its topography by moving the tip over the surface. To achieve this, two different types of positioning systems are required: one for the coarse motion to approach the tip very close to the sample, and a second one, with very high precision to scan the surface. The coarse actuator has to fulfill several requirements: large distances (in this system 300 $\mu \mathrm{m}$ ) have to be bridged, to retract the tip sufficiently for the sample transfer. On the other hand the movement still has to be precise enough to avoid crashing the tip into the surface while approaching it. Finally the vibrational stability should be as good as possible. There are several mature solutions for this engineering problem. In this system a Besocke type $[58,60]$ coarse motion is used. It has the advantage of allowing the tip to be placed on almost any spot in the $x-y$ plane of the sample, however the z-movement range is quite limited $(\sim 300 \mu \mathrm{m})$ so that very high precision for tip and sample mounting is required.

The fine positioning is done by a method that has proven itself in many previous STM designs [45,58]: a piezo tube, which is a $1 \mathrm{~cm}$ long hollow tube, with four segregated electrodes on the outside, and one on the inside. Depending on the voltages applied to different electrodes this piezo can be deformed in the $\mathrm{x}, \mathrm{y}, \mathrm{z}$ directions. A tip attached to one end of the tube will move accordingly over the surface. This positioning device delivers the required precision especially in the $\mathrm{z}$ direction, so that scanning the surface and detecting structures in the $\AA$ regime is possible. Figure 2.9 shows the piezo tube tip and the besocke ramp.

\section{Sample preparation facilities}

The system is equipped with a sputter gun in the preparation chamber. To achieve atomically clean metal surfaces the samples are bombarded with $\mathrm{Ar}+\mathrm{ion}$, which removes impurities and adsorbates. The increased surface roughness is then smoothed by annealing the sample with a oven that is mounted on the sample holder. This process has to be repeated a number of times to obtain a good sample surface. The overall quality of the surface can be checked with a LEED setup, before and after deposition of molecules/atoms etc. on the surface. Several evaporator slot are present and a wide range of samples can be prepared: A commercial triple ebeam metal evaporator can be used to grow thin metal films, a quadruple Kundsen cell evaporator deposits molecules, as well as several single evaporators for molecules and metals are available. It is further possible 
to deposit metal in-situ on the sample, because the cooling shields of the STM have an opening. The in-situ slot is also equipped with a fixed homebuild alkali evaporator, working with cells from SAES getters. The main manipulator of the chamber can be cooled with liquid nitrogen, so that the sample temperature during deposition can be chosen between $-180{ }^{\circ} \mathrm{C}$ to $500{ }^{\circ} \mathrm{C}$.

\subsection{Methods}

\subsubsection{Sample preparation}

In order to investigate the $\mathrm{MePc}(\mathrm{Me}=\mathrm{Fe}, \mathrm{Co}, \mathrm{Ni}, \mathrm{Cu})$ molecules adsorbed on the $\mathrm{Ag}(100)$ substrate we prepared several samples of varying molecular coverages. To avoid contaminations all experiments were undertaken under ultra high vacuum (UHV) conditions $\left(p<2 \cdot 10^{-10}\right.$ mbar). First we cleaned the $\mathrm{Ag}(100)$ surface by repeated sputtering with $\mathrm{Ag}^{+}$ions and subsequent annealing to $450{ }^{\circ} \mathrm{C}$. We monitored the surface quality by Low Energy Electron Diffraction (LEED). Once a clean surface was obtained the molecules were evaporated from the molecular beam source onto the sample, which was kept at room temperature. The Knudsen cell of the evaporator was heated to $440{ }^{\circ} \mathrm{C}^{\dagger}$, which yielded a deposition rate of approximately 0.05 molecular layers per minute. In this way samples of different MePc coverages could be produced, which were then transferred into the STM sample stage. To limit molecular mobility all measurements were done at $5 \mathrm{~K}$.

The doping experiments were prepared by in-situ deposition of Li or Fe atoms, as a second step after a MePc preparation. The source for the Li was a well degassed Li dispensor (SAES) heated by direct current flow (6 A), for the Fe atoms an electron beam evaporator was used.

The MePc molecules are commercially available from Sigma Aldrich in the form of a $99 \%$ pure powder. Before they can be used in an UHV environment, it is necessary to throughly degas the powder to achieve a base pressure during evaporation below $p<5 \cdot 10^{-10}$ mbar. The $\mathrm{Ag}(100)$ crystal was acquired from Surface Preparation Laboratories.

The $d I / d V$ spectra were obtained using the lock-in technique, using a bias voltage modulation of frequency around $3 \mathrm{kHz}$ and amplitude $1 \mathrm{mV}$

\footnotetext{
†This temperature is measured on the outside of the crucible close to the filament and therefore might not be the actual temperature of the molecules during the deposition.
} 
(rms) for the low range spectra, $3 \mathrm{mV}$ (rms) for the longer energy range and $10 \mathrm{mV}$ for $d I / d V$ maps.

\subsubsection{Density functional theory methods}

The spectroscopic results have been compared to the electronic structure obtained from ab-initio calculations performed by R. Robles, N. Lorente, R. Korytár, and P. Ordejón, using the VASP implementation of DFT in the projected augmented plane wave scheme [61,62]. Different approximations, each with different limitations and over- or underestimations have been compared in order to obtain consistent results, and to check the effect of different exchange-correlation approximations or the van der Waals (vdW) interaction. Namely we used Local Density Approximation (LDA) [63], Generalized Gradient Approximation (GGA) [64], GGA+vdW $[65,66]$, the DSRLL functional, designed to include $\operatorname{VdW}[67,68,69,70]$, and $\mathrm{LDA}+\mathrm{U}$ [71]. The plane wave cutoff energy was set to $300 \mathrm{eV}$. The calculated slab included $5 \mathrm{Ag}$ atomic layers intercalated by 7 vacuum layers in the vertical direction, and a $7 \times 7$ lateral supercell. The positions of all atoms in the molecule and the first three $\mathrm{Ag}$ layers were relaxed vertically and laterally until forces were smaller than $0.05 \mathrm{eV} / \AA$. The Projected Density of States (PDOS) of NiPc have been used to compare single $(1 \times 1 \times 1)$ and multiple $(5 \times 5 \times 1) k$ point calculations, which converge after applying a broadening of $100 \mathrm{meV}$ to the data. Based on that, the results for a single $k$ point with the latter broadening are used in the following. Charge transfer and local magnetic moments have been calculated using a Bader charge analysis [72, 73]. 


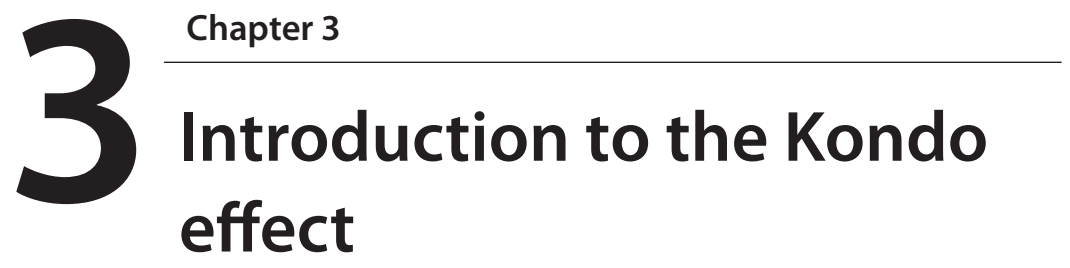

Conduction measurements of magnetic systems at low temperature, like the present study of $3 \mathrm{~d}$ transition metal $\mathrm{MePc}$ on $\mathrm{Ag}(100)$ are closely intertwined with a phenomenon known as the Kondo effect. Essentially it is a coupling of the conduction electrons of a metal to a magnetic impurity. Below a critical temperature, the so-called Kondo temperature, the coupling leads to a screening of the spin of the magnetic impurity due to the creation of a many body singlet state. This gives rise to a typical sharp resonance in the DOS at the Fermi level, which can be accessed experimentally by STS measurements. This Kondo resonance is characteristic of a magnetic impurities on a non-magnetic surface. Hereby it provides a method for STM measurements to probe the magnetic properties of certain adatoms or molecules.

As the Kondo effect is intrinsically a many-body problem, its mathematical description is necessarily complex. Since its first explanation in the 1960, many advanced mathematical tools such as the Numerical Renormalization Group (NRG), and the Bethe ansatz have been applied to solve the problem. In this chapter we present an outline of the basic concepts in a descriptive manner, in order to understand the mechanisms involved. For a more complete treatment the reader is referred to specific literature [74, 75, 76, 77]. 


\subsection{The Kondo problem}

In the 1930s measurements of the electrical resistivity of certain metals revealed an effect that would puzzle physicists for 30 years [78]: as the temperature of the sample is lowered, the resistivity reaches a minimum and then increases again as $-\ln (T)$, for even lower temperature (see Figure 3.1). Later it was found that this effect only occurs if the metal contains dilute magnetic impurities, such as iron or cobalt atoms.

In metals, the electric resistivity is caused by the scattering of conduction electrons. They can scatter of crystal lattice vibrations (phonos) or of static defects in the lattice itself such as vacancies or substitutions.

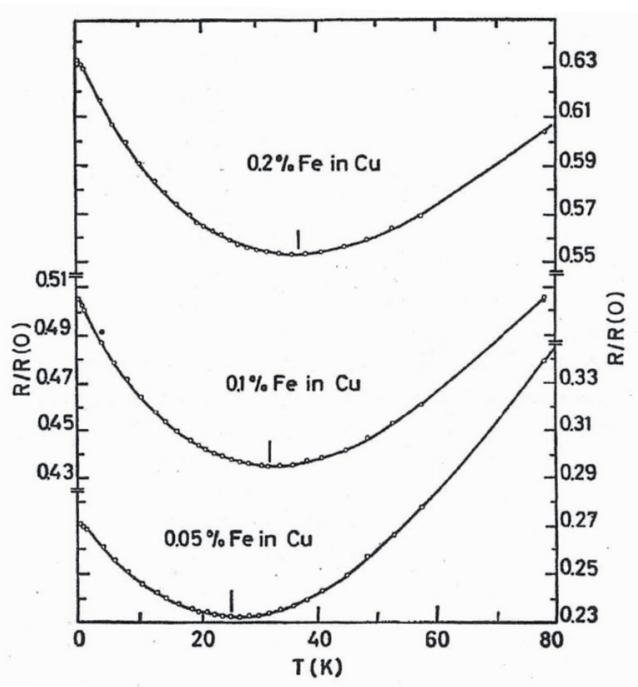

Figure 3.1.: Resistivity of a copper crystal, with different percentages of Fe impurities. Figure adapted from [79].

As the temperature decreases, lattice vibrations become less pronounced, hence the effect of phonon scattering on the resistivity goes down $\left(\propto T^{5}\right)$. The contribution of the static impurities remains unchanged, and the resistivity saturates at a minimum caused by these defects. 
The increase in resistivity observed for the dilute magnetic alloys was explained by J. Kondo in 1964 [80], by introducing another scattering mechanism for the conduction electrons. The spin flip interaction between the magnetic impurities and the spin of the conduction electrons. He described this in the s-d exchange model or Kondo model:

$$
\begin{array}{r}
H=H_{\text {Bloch }}+H_{K} \\
H_{K}=-J \vec{S} \cdot \vec{s}(r)
\end{array}
$$

The Hamiltonian consists of a $H_{B l o c h}$ part describing the non interacting electrons in a Bloch model, and the coupling part $H_{K}$ which couples the spin $\vec{S}$ of the impurity to the spin $\vec{s}$ of the conduction electrons at the site of the impurity $r$. A Heisenberg type spin interaction is represented by $J$. Using this Hamiltonian in third order perturbation theory to determine the resistivity, J. Kondo was able to reproduce the $-\ln (T)$ behavior observed in the dilute alloys. We will not go into details, because the calculations are rather lengthy and do not provide any further insight into the physics involved.

There are however several important details to point out: Within the Kondo model $J$ is assumed to be constant and positive, implying antiferromagnetic coupling between the impurity and the conduction electrons. This coupling sign is a necessary condition for the perturbation theory to produce the correct results. A more physical explanation can be found in the Schriefer-Wolff transformation [81] (see section 3.3 on page 40).

The Kondo model, however, has some limitations. The description of the impurity as a spin $\frac{1}{2}$ system, is with regard to real impurities an insufficient description. Higher spin or more complex mixed charge states for impurities are possible. A more problematic shortcoming is the breakdown of Kondo's model for very low temperatures. Below a critical temperature $T_{K}$, it makes the unphysical prediction that the resistivity will be infinite for $T<T_{K}$. This critical temperature became known as the Kondo temperature:

$$
k_{B} T_{K}=D e^{-1 / 2 J \rho_{o}}
$$

Where $D$ is the width and $\rho_{o}$ the density of states of the conduction band. One has to be careful not to confuse the temperature of the 
resistivity minimum with this temperature. When the system reaches the Kondo temperature, the magnetic properties of the impurity start changing. As will be detailed later in section 3.4, its moment is screened by the conduction electrons, forming a many body singlet state.

The first solution to the Kondo problem was found by Anderson using a scaling approach, known as "poor man's scaling", which gives a qualitative understanding of the Kondo problem [82]. A theory that produced quantitative results is the "numerical renormalization group" method developed by Wilson in the mid 70ties [83]. The discussion of these theories lies beyond the scope of this work, instead we will follow a more descriptive approach.

\subsection{The Anderson model}

Kondo's spin flip scattering model for dilute alloys focuses on the phenomenological description of the resistivity minimum. A microscopic model describing a magnetic impurity inside a conducting metal is the Anderson Model [84]. It offers a very physical description, and thus helps to understand the underlying mechanism involved. The central question it tries to answer is whether a magnetic impurity interacting with a host metal retains is magnetic character or not. The system is modeled by the use of only three parameters, which makes it rather easy to handle as numerical model. It was shown by Schrieffer and Wolf that it is possible to transform Anderson's into Kondo's Hamiltonian, and that for certain parameters the Anderson model describes the same physics as the Kondo model (see section 3.3 on page 40 ).

\section{The Anderson Hamiltonian}

The Anderson model reduces the problem of a dilute magnetic alloy to a single magnetic impurity placed in a non magnetic host metal (see Figure 3.2). A magnetic atom would have unpaired electrons, e.g., in $3 \mathrm{~d}$ or $4 \mathrm{f}$ states, which are represented as a single impurity state $E_{d}$, which can be either singly or doubly occupied. These states are quite localized, therefore the addition of an extra electron has to overcome a strong Coulomb repulsion. This behavior is modeled by introducing an additional potential $U$, reducing the probability of double occupation. As we shall see later this term plays a very important role in the creation of a magnetic moment in 


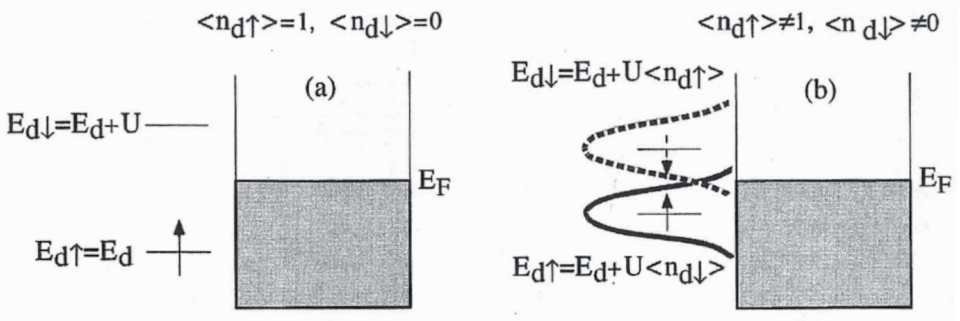

Figure 3.2.: A schematic view of the Anderson model. The impurity is modeled as a singly occupied localized state, double occupation is inhibited by an on-site Coulomb repulsion $U$. The metal is a continuum of states filled up to $E_{F}$. a) The hybridization of the local levels is neglected, while the Coulomb repulsion is already considered. b) The complete Anderson model: the hybridization is switched on leading to a broadening of the impurity states, which to some extent equalizes the occupancy of spin up and spin down level. Image adapted from [76].

the impurity. The formal Hamiltonian Equation 3.4 is then constructed as follows. The host metal is represented as non interacting Bloch electrons, the local impurity state is modeled as extra localized orbitals, idealized as one state with two possible spin orientations, any degeneracy of its orbitals is neglected. Another term describes the hybridization between the conduction band electrons and the impurity:

$$
\begin{aligned}
H_{A} & =\underbrace{\sum_{\vec{k} \sigma} E_{k} n_{\vec{k} \sigma}}_{\text {conduction band }}+\underbrace{\frac{1}{\sqrt{N}} \sum_{\vec{k} \sigma}\left[V_{\vec{k} d} c_{\vec{k} \sigma}^{\dagger} c_{d \sigma}+V_{\vec{k} d}^{*} c_{d \sigma}^{\dagger} c_{\vec{k} \sigma}\right]}_{\text {hybridization }} \\
+ & \underbrace{E_{d}\left(n_{d \uparrow}+n_{d \downarrow}\right)}_{\text {impurity state }}+\underbrace{U n_{d \uparrow} n_{d \downarrow}}_{\text {coulomb repulsion }}
\end{aligned}
$$

where $E_{k}$ is the energy level of the conduction band state, $n_{\vec{k} \sigma}=c_{\vec{k} \sigma}^{\dagger} c_{\vec{k} \sigma}$ is the electron number operator, while $c_{\vec{k} \sigma}^{\dagger}, c_{\vec{k} \sigma}$ refer to the respective creation and annihilation operators. $E_{d}$ is the impurity state energy with $n_{d \uparrow}$ and $n_{d \downarrow}$ again the number operators for the impurity orbital (with $c_{d \sigma}^{\dagger}, c_{d \sigma}$ creation and annihilation operators). The hybridization term 
depends on the transition amplitude $V_{\vec{k} \sigma}$ and is normalized by the number units cells of the host metal $N$. The strength of the Coulomb repulsion is denoted as $U>0$.

The behavior of the impurity is thus determined by three parameters:

- the on-site Coulomb repulsion $U$ at the impurity, which favors a singly occupied impurity orbital and thereby a persisting moment.

- the position of the impurity energy level $E_{d}$ with respect to the Fermi energy $E_{F}$ of the host metal. If $E_{d}<E_{F}$ and $E_{d}+U>E_{F}$ the impurity level will be singly occupied, while $E_{d}<E_{d}+U<E_{F}$ would lead to a doubly occupied impurity level.

- the hybridization between the local and conduction states causing a quenching of the moment by smearing out the occupation of the impurity level, as we shall see in the next section.

While the effects of the two first parameters are rather straightforward to identify, the hybridization requires more consideration. From an intuitive point of view the consequence of hybridization between a localized state $E_{d}$ and the continuum leads to a broadening of the former impurity levels. Indeed the treatment of the Anderson Hamiltonian leads to a Lorentzian line shape for the density of states of the impurity levels, with the broadening depending on the hybridization term $V_{\vec{k} \sigma}$.

Using a Green's function $G^{0}(E)=\left(E-H_{A}\right)^{-1}$ approach and a HarteeFock approximation to simplify the operator $U n_{d \uparrow} n_{d \downarrow} \approx U n_{d \uparrow}\left\langle n_{d \downarrow}\right\rangle+$ $U n_{d \downarrow}\left\langle n_{d \uparrow}\right\rangle$ one finds the occupation of the impurity levels to be [84]:

$$
\begin{gathered}
n_{d \uparrow(\downarrow)}(E)=\frac{1}{\pi} \frac{\Gamma}{\left(E-E_{d}-U\left\langle n_{d \downarrow(\uparrow)}\right\rangle\right)^{2}+\Gamma^{2}} \\
\text { with } \Gamma=\pi\left|V_{\vec{k} \sigma}\right|^{2} n_{o}\left(E_{F}\right)
\end{gathered}
$$

The term $n_{o}\left(E_{F}\right)$ denotes the electronic density of states of the host at the Fermi-level for a given spin direction per unit cell. $\Gamma$ will be the parameter characterizing the hybridization.

Using these terms to integrate the density of states up to the Fermi-level, the occupation of the impurity level can be found: 


$$
\begin{aligned}
\left\langle n_{d \downarrow}\right\rangle & =\int_{-\infty}^{E_{F}} n_{d \uparrow}(E) d E \\
\left\langle n_{d \uparrow}\right\rangle & =\int_{-\infty}^{E_{F}} n_{d \downarrow}(E) d E
\end{aligned}
$$

The solutions have to be determined graphically, because the resulting equations (Equation 3.6 and Equation 3.7) are still coupled. Note that, if $\Gamma \gg U$ only non-magnetic solutions $\left\langle n_{d \downarrow}\right\rangle=\left\langle n_{d \uparrow}\right\rangle=\frac{1}{2}$ appear. Equally if $\Gamma \ll U$ only the magnetic solution is found $\left\langle n_{d \uparrow}\right\rangle \approx 1,\left\langle n_{d \downarrow}\right\rangle \approx 0$. The transition between the magnetic and non-magnetic solution occurs at $\pi \Gamma=U$. Finally one obtains a phase diagram showing all magnetic and non magnetic solutions for different sets of parameters for the Anderson Hamiltonian, shown in Figure 3.3.

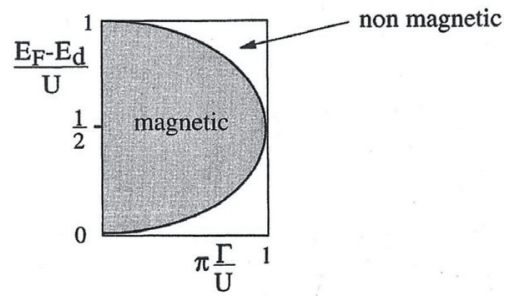

Figure 3.3.: Phase diagram showing all the magnetic and nonmagnetic states as predicted by the Hartree Fock treatment of the Anderson model. Image adapted from [84]

In conclusion the Anderson model describes the stability of a magnetic impurity in a non magnetic host metal. It includes two types of counteracting potentials: first the on site Coulomb repulsion which leads to singly occupied impurity state, which maintains the magnetic moment. Counteracting this effect is the hybridization between the impurity state and the conduction electrons, smearing out the occupation and effectively reducing the magnetic moment.

It is important to note that all these considerations are only valid at high enough temperatures $T>T_{K}$. As the thermal energy of the systems is lowered, any remaining magnetic moment will be screened by strong electron correlation effects, i.e., the Kondo effect (See section 3.4 on page 42). 


\subsection{Virtual spin flips and the Schrieffer - Wolff transformation}

As just described in section 3.2 the Anderson model predicts that the local moment of a magnetic impurity persists if the on-site Coulomb repulsion is larger than the hybridization energy. In this case it essentially describes the same physics as the Kondo model. Schrieffer and Wolff have shown that it is possible to obtain the Kondo Hamiltonian from the Anderson model, in its magnetic limit, by diagonalizing it in the subspace of singly occupied impurity states [81] . This is called the Schrieffer Wolff transformation.

Before entering into details, we would like to present an intuitive way of picturing the Kondo model in terms of the Anderson model, i.e., the Schrieffer Wolff transformation. Consider the following situation in the Anderson model: the impurity level $E_{d}$ lies well below $E_{F}$ and the Coulomb repulsion counterpart above the Fermi level $\left(E_{d}+U>E_{F}\right)$. The temperature is sufficiently low to exclude thermal excitations, $k_{B} T \ll\left|E_{d}\right|$, $E_{d}+U$. This situation leads to a singly occupied impurity level. As the energy of the system is low, no classical exchange of the impurity and the conduction spin is possible. However quantum mechanics permits two virtual excitations that can flip the spin of the impurity: In Figure 3.4a) one electron tunnels from the magnetic atom to the conduction band to a state near the Fermi level, which are the only ones available due to the low temperature. Another electron tunnels back into the impurity. The new electron can now have its spin oriented the other way, thus the impurity spin is effectively flipped without any energy cost. Applying the same arguments another spin flip process is possible, shown in Figure 3.4b). An electron from the conduction band tunnels into the impurity, temporarily doubly occupying it. According to the Pauli principle the spin of incoming electron has to be opposite to the one of the impurity electron. If now the original localized electron tunnels out, the impurity spin is flipped again.

This intuitive reasoning outlines how, at low temperature, the Anderson model can be transformed into a model that considers only one effective spin in the impurity, which can be flipped by virtual processes with the conduction electrons. Hence in the case of a singly occupied level, the impurity can be described by its spin $\vec{S}$. The coupling to the spin $\vec{s}$ of the conduction electrons is proportional to a coupling constant J. The interaction is thus found to be $J \vec{S} \vec{s}$, which is exactly the Kondo model. 
a)
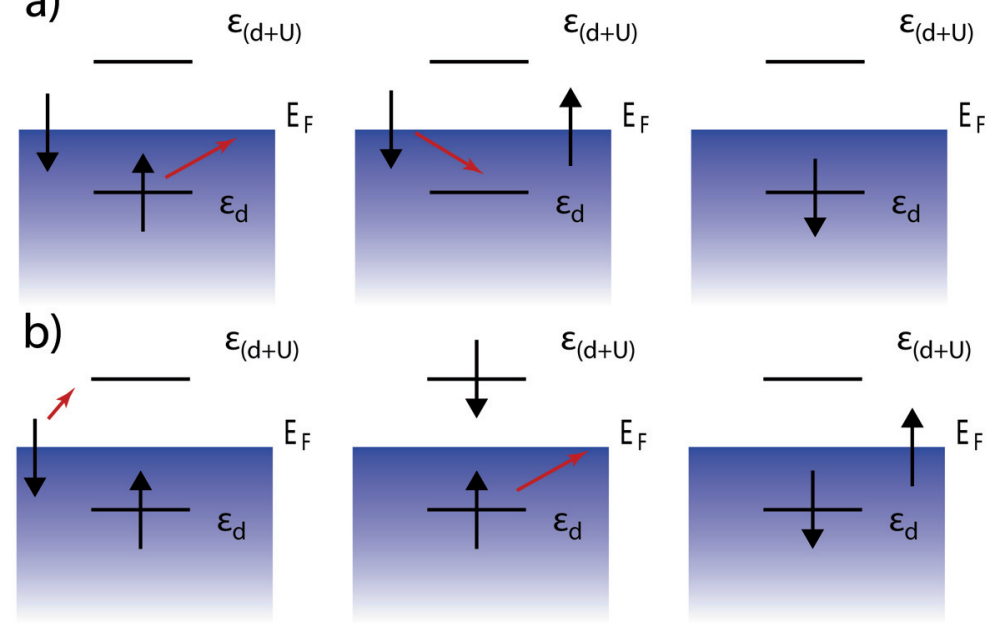

Figure 3.4.: Two processes that contribute to the second order fluctuations on the impurity. a) The conduction electron tunnels through the hybridized level into the impurity, creating a double occupied intermediate state. Afterwards the original impurity electron leaves the impurity. b) The impurity electron leaves and is then replaced by a conduction electron. $\mathrm{U}$ is the Coulomb repulsion, $d$ is the impurity level. Image adapted from [77].

The actual mathematical Schrieffer-Wolff transformation of the Anderson to Kondo Hamiltonian is a canonical transformation in lowest order [77]. It predicts the sign of the coupling constant J to be antiferromagnetic, which in Kondo's model was a parameter.

The mathematical form of the transformation S can be written as [81]: 


$$
S=\sum_{k, \sigma, \alpha= \pm} \frac{V_{k, d}}{\epsilon_{k}-\epsilon_{\alpha}} n_{d,-\sigma}^{\alpha} c_{k . \sigma}^{\dagger} d_{\sigma}-H . C .
$$

with :

$$
\begin{gathered}
\epsilon_{+}=\epsilon_{d}+U \\
\epsilon_{-}=\epsilon_{d} \\
n_{d,-\sigma}^{+}=n_{d,-\sigma} \\
n_{d,-\sigma}^{-}=1-n_{d,-\sigma} \\
H_{A}=H_{0}+H_{1} \\
H_{S W}=H_{0}+\frac{1}{2}\left[S, H_{1}\right]
\end{gathered}
$$

Where $H_{S W}$ denotes the transformed Hamiltonian, $H_{1}$ the perturbed part of the Anderson Hamiltonian, which is the hybridization of the local level and the conduction band. $H_{0}$ is the rest of the Anderson model, i.e., the conduction band, the Coulomb repulsion and the localized level. When comparing Equation 3.10 to Equation 3.1 we see that the two Hamiltonians are in essence the same.

Through this approach it can be shown that the coupling constant $J$ is given by [77]:

$$
J \approx \frac{U}{\left|E_{d}\right|\left(U-\left|E_{d}\right|\right)}<0
$$

Thus charge fluctuations, i.e., virtual excitations in the Anderson model at low temperature lead to a spin dependence of the interaction. The Pauli principle is the reason for this, as it forbids to doubly occupy the impurity with electrons of the same spin. We have seen, how the transformed Anderson model leads to a Kondo model with an anti-ferromagnetic exchange coupling, resulting in the condensation of the system in a singlet state, as will be discussed in the next section.

\subsection{Formation of the Kondo singlet}

Experiments on dilute magnetic alloy revealed yet another "anomalous" behavior in systems with a resistivity minimum. The magnetic susceptibility ceases to follow Curie's law and saturates to a constant value for 
$T=0$, indicating that the magnetic moment is completely quenched at low temperature. This behavior can be explained by the formation of a many body singlet state is formed by the impurity and the surrounding conduction electrons at the Fermi energy.

\subsubsection{The spin $\frac{1}{2}$ Kondo effect}

\section{A molecular model}

The screening of the local moment is already included in the Anderson model. The mechanism involved can be nicely understood in a simplified description as a two orbital molecule [76]. We make an extreme simplification by treating the host metal as just one extended $\left(\epsilon_{k}\right)$ orbital and the impurity as a single localized orbital $\left(\epsilon_{f}\right)$. We use an Anderson type Hamiltonian, and consider two electrons inside the molecule. The parameter of the model are: the Coulomb repulsion $U$ for the localized orbital and the hybridization parameter $V_{s f}$. In the case of zero Coulomb repulsion $(U=0)$ this model describes the two orbitals with the energies $\epsilon_{k}$ and $\epsilon_{f}$ interacting with the off diagonal element $V_{s f}$.

$$
\left|\begin{array}{cc}
\epsilon_{k}-E & V_{s f} \\
V_{s f} & \epsilon_{f}-E
\end{array}\right|=0
$$

The diagonalization of this Anderson Hamiltonian leads to bonding and anti-bonding states with the energies $E_{a}$ and $E_{b}$. The hybridization between the two orbitals is assumed to be small $\left|V_{s f}\right| \ll \epsilon_{k}-\epsilon_{f}=\Delta$ thus the energies will be respectively:

$$
E_{b}=\epsilon_{f}-\frac{\left|V_{s f}\right|^{2}}{\Delta} \quad E_{a}=\epsilon_{k}+\frac{\left|V_{s f}\right|^{2}}{\Delta}
$$

The two electrons can now be distributed in six different ways into these two orbitals, creating one triplet $(S=1)$ and three singlet $(S=0)$ states, as shown in Figure 3.5. The ground state will the configuration with 2 electrons in the bonding orbital forming a singlet state.

If the on-site repulsion is switched on, and for the sake of simplicity we use $U=\infty$, the energy landscape changes significantly. There are five possible distributions for the electrons in the two orbitals, because the double occupation of the localized orbital is forbidden (see Figure 3.6).

Two singlet states can be formed : 


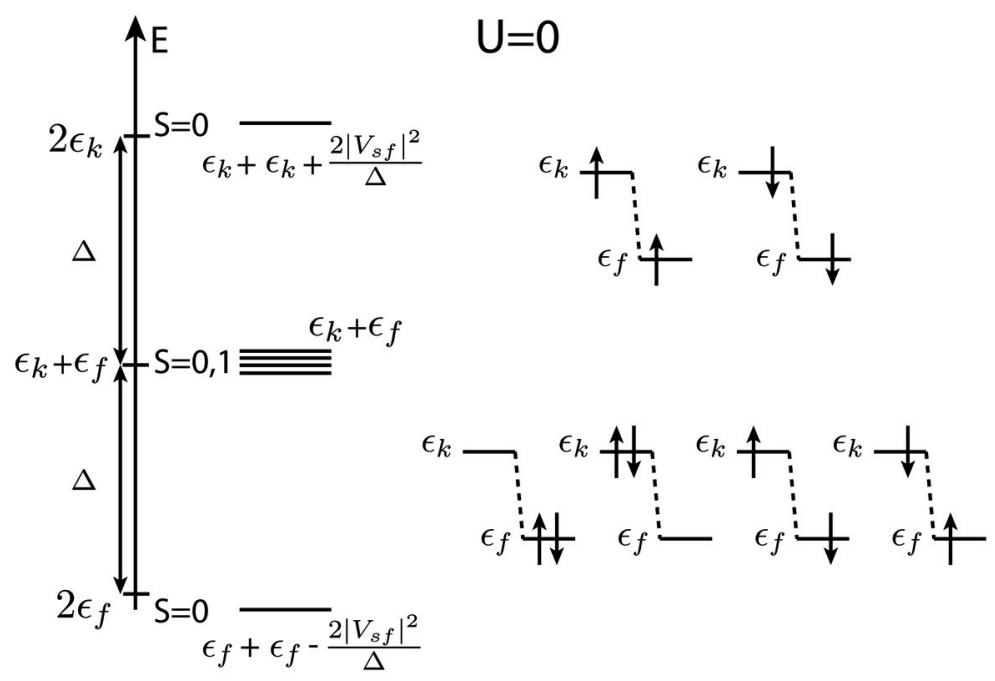

Figure 3.5.: The energy levels of the molecular model, for the case of the on site Coulomb repulsion being $U=0 . \epsilon_{k}$ stands for the extended orbital (a crude model of the conduction band), while $\epsilon_{f}$ for the localized orbital (i.e. the impurity), hybridization is assumed to be small $\left|V_{s f}\right| \ll \epsilon_{k}-\epsilon_{f}=\Delta$. The possible two-electron occupation configurations are shown on the right allowing the creation of one triplet and three singlet states.

$\psi_{1}^{(S=0)}=c_{k \uparrow}^{\dagger} c_{k \downarrow}^{\dagger}|0\rangle \quad, \quad \psi_{2}^{(S=0)}=\frac{1}{\sqrt{2}}\left[c_{k \uparrow}^{\dagger} c_{f \downarrow}^{\dagger}-c_{k \downarrow}^{\dagger} c_{f \uparrow}^{\dagger}\right]|0\rangle$

with the corresponding Hamiltonian :

$$
H_{m}=\left(\begin{array}{cc}
2 \epsilon_{k} & \sqrt{2} V_{s f} \\
\sqrt{2} V_{s f}^{*} & \epsilon_{k}+\epsilon_{f}
\end{array}\right)
$$

giving the eigenvalues:

$$
E=\epsilon_{k}+\frac{1}{2}\left[\epsilon_{k}+\epsilon_{f} \pm \sqrt{\left(\epsilon_{k}-\epsilon_{f}\right)^{2}+8\left|V_{s f}\right|^{2}}\right]
$$


which then by a series expansion and the assumption $\left|V_{s f}\right| \ll \epsilon_{k}-\epsilon_{f}=$ $\Delta$ can be written as:

$$
E^{(S=0)}=\epsilon_{k}+\epsilon_{f}-\frac{2\left|V_{s f}\right|^{2}}{\Delta} \quad E^{(S=0)}=\epsilon_{k}+\epsilon_{k}+\frac{2\left|V_{s f}\right|^{2}}{\Delta}
$$

One triplet can also be formed, with the wavefunctions for $S_{z}=1,0,-1$ :

$$
c_{k \uparrow}^{\dagger} c_{f \uparrow}^{\dagger}|0\rangle \quad, \quad \frac{1}{\sqrt{2}}\left[c_{k \uparrow}^{\dagger} c_{f \downarrow}^{\dagger}+c_{k \downarrow}^{\dagger} c_{f \uparrow}^{\dagger}\right]|0\rangle \quad, \quad c_{k \downarrow}^{\dagger} c_{f \downarrow}^{\dagger}|0\rangle
$$

The energy of the triplet state is accordingly:

$$
E^{(S=0)}=\epsilon_{k}+\epsilon_{f}
$$

By combining Equation 3.17 and Equation 3.18 we find the energy landscape (see Figure 3.6):

$$
\begin{aligned}
E^{(S=0)} & =\epsilon_{k}+\epsilon_{f}-\frac{2\left|V_{s f}\right|^{2}}{\Delta}<E^{(S=1)}=\epsilon_{k}+\epsilon_{f} \\
& <E^{(S=0)}=\epsilon_{k}+\epsilon_{k}+\frac{2\left|V_{s f}\right|^{2}}{\Delta}
\end{aligned}
$$

It becomes clear that the ground state of the system is a singlet state, which lies lower in energy than the triplet. The spin of the localized level is effectively screened. The energy difference between them $\frac{2\left|V_{s f}\right|^{2}}{\Delta}$ however is rather small, it corresponds to one spin flip to form the triplet state. If the thermal energy of the system is high enough, i.e., above $k_{B} T_{K}=\frac{2\left|V_{s f}\right|^{2}}{\Delta}$ these flips can be pictured as spin fluctuations. Hence as the temperature is lowered the spin fluctuations between the ground singlet and the triplet state cease to occur and the singlet state becomes predominant.

We have found this result using a dramatically simplified description of the conduction band, however the mechanism for the formation of the singlet ground state is in essence the same when a complete band is considered. There is one very important difference however: the true Kondo ground state is not formed by the impurity electron and a single conduction electron. The screening of the impurity moment is many body effect, involving all conduction electrons close to the Fermi energy. 


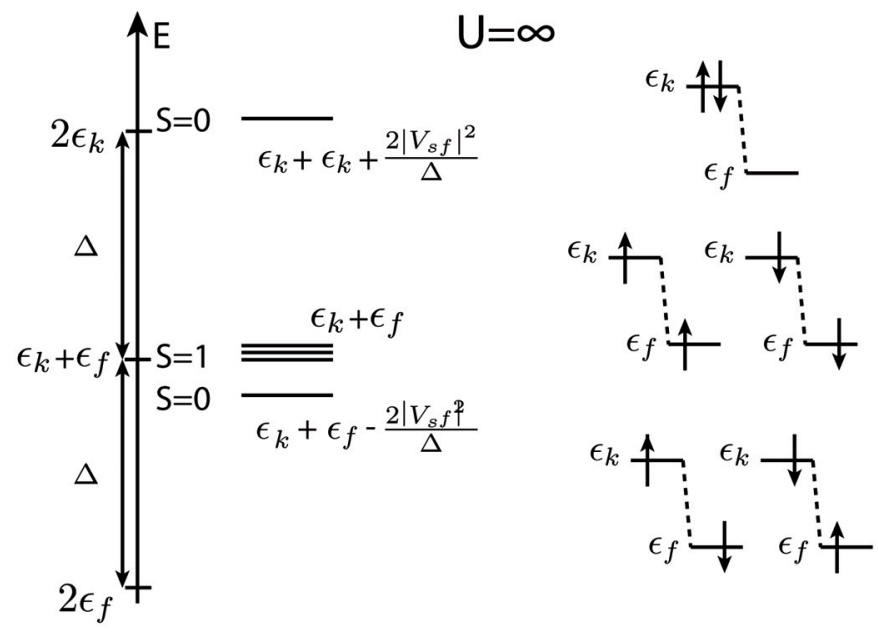

Figure 3.6.: The energy levels of the molecular model, for the case of the on site Coulomb repulsion being $U=\infty$. The possible two electron occupation configurations are shown on the right for the molecular model in the limit $U=\infty$, thus double occupation for the impurity $\epsilon_{f}$ is not possible, allowing the creation of one triplet and two singlet states.

\section{The Kondo Temperature}

In light of this model we can now better understand the Kondo temperature, which earlier was defined as the breakdown of the Kondo model (Equation 3.3). The molecular model showed that below a certain temperature the magnetic moment is screened because the system can lower its energy by forming a singlet state. This state breaks up if the thermal energy of the system is higher than the energy gained by its formation. The Kondo temperature is defined as the energy gained by the system through the formation of the Kondo singlet. Exact treatment of the Anderson model with an advanced mathematical method like the NRG finds the following expression [85]:

$$
T_{K}=\frac{1}{2}(\Gamma U)^{2} \exp \left(\frac{\pi E_{d}\left(E_{d+U}\right)}{\Gamma U}\right)
$$


were $\Gamma$ is the width of the impurity energy level $E_{d}$ and $U$ the on-site Coulomb repulsion.

\section{The zero bias Kondo resonance}

The formation of the singlet state has another consequence for the system, as first noticed by Abrikosov[86] and Suhl [87]: a very sharp resonance appears in the DOS exactly at the Fermi level, its half width at $T=0$ is $k_{B} T_{K}$.

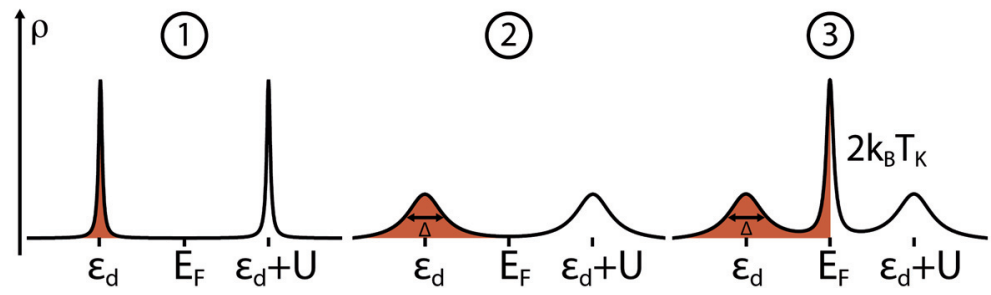

Figure 3.7.: Density of states in the Anderson model: 1) Only the impurity levels. 2) The impurity interacting with the conduction sea well above the Kondo temperature. 3) and well below the Kondo temperature. The many body singlet causes the sharp peak at the Fermi energy.

In the complete Anderson model a tangible qualitative picture can be created: The host electrons, with an energy $E=k_{B} T_{K}$ around $E_{F}$ constantly tunnel in and out of the impurity state along the lines of the argumentation for the Schrieffer Wolff transformation, creating a new quasi particle state. This can be thought of as a cloud of electrons, whose spin is correlated due to the interaction with the impurity spin and the Pauli principle. Since this spin flip mechanism does not require any energy, the resonance is pinned at the Fermi level. Its width $\Gamma_{K}$ depends on the Kondo temperature as well as the measurement temperature, and follows an expression derived from Fermi liquid theory [88, 89]:

$$
\Gamma_{K}(T)=2 \sqrt{\left(\pi k_{B} T\right)^{2}+2\left(k_{B} T_{K}\right)^{2}} .
$$

\subsubsection{The underscreened Kondo effect}

So far we have considered impurities with a total spin of $S=1 / 2$, which at temperatures far below $T_{K}$ are completely screened by the conduction 
electrons. What happens, however, when the total spin is greater than $1 / 2$, e.g., for a $\mathrm{S}=1$ system?

The behavior of such a system depends on the number of screening channels and on its temperature. The overlap of the wave functions of the impurity and the conduction electrons leads to the creation of screening channels. If the number $n$ of channels is high enough $(n=2 S)$, even large magnetic moments will be completely screened [90].

Consider an $S=1$ impurity with two screening channels, which are different due to different overlap with the conduction electrons. Both channels will have their own antiferromagnetic Kondo coupling constant $J_{1,2}$, which depend on the hybridization $\Gamma$ with the conduction electrons. Consequently Equation 3.3 will give two Kondo temperatures $T_{K_{1}}$ and $T_{K_{2}}$. The coupling is asymmetric, so that one Kondo temperature will be larger than the other $T_{K_{1}}<T_{K_{2}}$. The behavior of such a system, then depends on the temperature. If $T \ll T_{K_{1}}<T_{K_{2}}$ the spin will we completely screened, a situation known as a two-stage Kondo effect. An underscreened scenario occurs for $T_{K_{1}}<T<T_{K_{2}}$. The spin of the impurity will be only partially screened form $S=1$ to $S=1 / 2$, because only one channel is "active".

This situation has an impact on the intensity $G_{K}$ of the Kondo resonance as a function of temperature. For the fully screened Kondo effect, the conductance increases logarithmically above the Kondo temperature and below it, saturates in a quadratic fashion. On the other hand for the underscreened Kondo effect two different logarithmic behaviors above and below the higher Kondo temperature $T_{K_{2}}$ occur [91, 92, 93]. This is best seen by plotting the derivative of conductance with respect to temperature as a function of inverse temperature. In Figure 3.8 the behavior of the intensity of the Kondo resonance for a normal and an underscreened process is depicted.

The temperature dependent conductance obtained by numerical renormalization group (NRG) can be described using the following empirical formula $[25,91]$ :

$$
G_{K}(T)=G_{\text {off }}+G(0) \cdot\left[1+\left(\frac{T}{T_{K}}\right)^{\xi} \cdot\left(2^{1 / \alpha}-1\right)\right]^{-\alpha}
$$

where $\Gamma_{K}$ is the width (FWHM) and $G_{K}$ the intensity of the Kondo resonance, $\alpha$ and $\xi$ are parameters that depend on the total spin of the 
molecule (see Table 3.1).

\begin{tabular}{ccc}
\hline spin & $\xi$ & $\alpha$ \\
\hline $\mathrm{S}=1 / 2$ & 2 & $0.220 \pm 0.005$ \\
$\mathrm{~S}=1$ & $0.745 \pm 0.009$ & $0.506 \pm 0.009$ \\
\hline
\end{tabular}

Table 3.1.: Fitted parameters determined for Equation 3.22 to approximate the NRG predictions for the temperature dependence of the Kondo resonance for an impurity with spin $\mathrm{S}$ and a single screening channel. Adapted from [25].

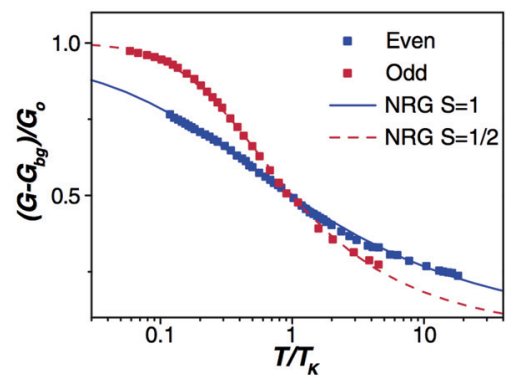

Figure 3.8.: Intensity of the Kondo resonance versus the inverse temperature recorded for a $\mathrm{C}_{60}$ molecule coupled to two metallics leads. The electron occupation of the molecule can be changed from odd to even leading to different spin states, with a fully screened and an underscreened Kondo effect. The curves are fitted with Equation 3.22. Adapted from [91].

Examples that show this kind of behavior are a $\mathrm{C}_{60}$ molecule placed off centered between two metallic leads, with a different coupling constant for each of them [91], Co(tpy-SH)2 complexes in break-junction experiments [25], or molecules adsorbed on an surface, where two magnetic moments are differently coupled to the surface electrons (this work).

\subsubsection{The inelastic Kondo effect}

So far we have discussed the equilibrium Kondo effect, which leads to a sharp resonance at zero-bias. It is however possible for the Kondo interaction to couple to inelastic excitations. The coupling to interorbital, vibrational [95, 96, 97, 94], magnetic excitations [98, 99, 100, 25, 101], photon adsorption/emission [102], cooper pair formation [103] has been observed. In molecules, like the ones investigated in this work only the coupling to vibrational, and magnetic excitation persist, due to the electronic level spacing of $\Delta E \gg T_{K}$, and the absence of light. 

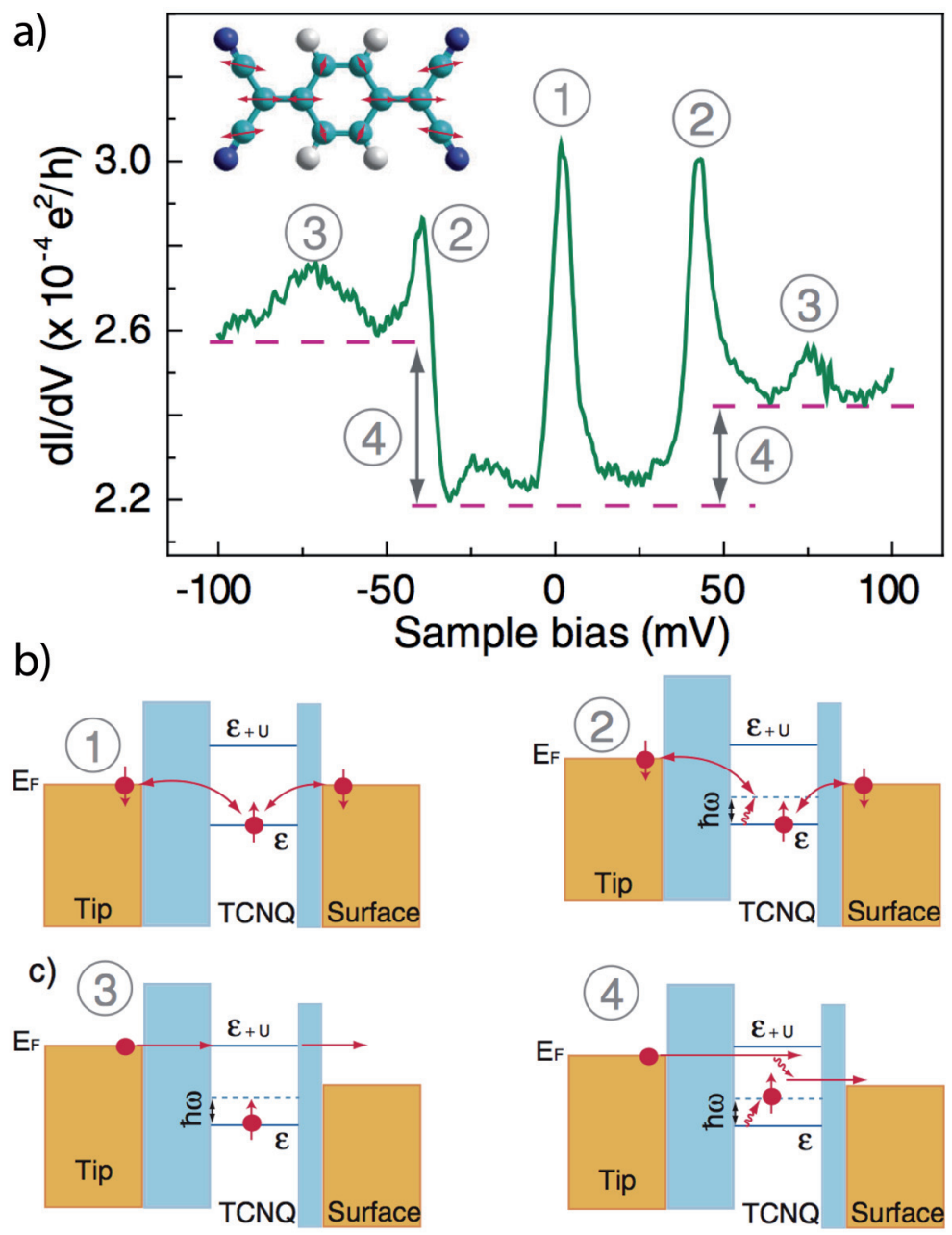

Figure 3.9.: $\mathrm{A} \mathrm{dl} / \mathrm{dV}$ spectrum taken on the center of a tetracyanoquinodimethane TCNQ molecule adsorbed on Au(111). Various elastic and inelastic processes can be identified. The inset shows the motion of TCNQ carbon atoms for the 9 vibrational mode. The arrows are proportional to the amplitude of motion. b) Schematics of the Kondo (1) and the phononassisted inelastic Kondo (2) tunneling, observed as sharp peaks in the spectrum a). Energy diagram of resonant tunneling through the doubly occupied LUMO state (3) and inelastic (off-resonance) tunneling (4), observed as broad peaks and steps in the spectrum (a), respectively. Similar diagrams can be constructed for negative sample bias. Image adapted from [94]. 
Depending on the molecular orbital, the electrons can couple to the vibrations of the molecule while tunneling through it. This is a similar process to the one described in the context of IETS (see subsection 2.2.2 on page 20): electrons tunneling through molecular orbitals can couple to molecular vibration directly. For the Kondo effect, the basic principle can be understood in the virtual spin flip picture described on page 40 . An excited electron or hole tunnels into the impurity, but at the same time emits a phonon. These processes will create two additional sidebands above and below $E_{F}$ at the energy of the excited phonon [95]. One has to bear in mind that these kind of features will be superposed to the normal inelastic coupling to phonons, which gives rise to step like conduction features, and of course the actual impurity level (see Figure 3.9 for an example).

Magnetic excitations occur in much the same way. For instance in a system with a singlet ground state, the tunneling electrons can induce spin-flip tunneling when the applied bias is large enough to induce transitions to triplet states. Such processes give rise to Kondo resonance peaks out of equilibrium [100].

\subsection{The Kondo effect in STM measurements}

In recent years scientific interest in the Kondo effect has been renewed. Thanks to new experimental techniques, it became possible to study Kondo systems at the nanoscale, for instance in single atoms or molecules. Break-junction, quantum dot and STM measurements allow an unprecedented control over physical parameters, and have led to the discovery of many new forms of Kondo behavior, inaccessible in bulk studies of diluted alloys. An extensive review of these devices is given in Refs. [104, 105], here we will present a few examples of STM studies.

STM measurements of Kondo system allow access to real space images of Kondo resonances, providing the possibility to investigate the effect directly for a single atom, while at the same time being able to manipulate the structure on the surface, investigating the relationship between geometrical arrangements, chemical bonding, and the Kondo interaction. Measurements of single magnetic adatoms on noble metal surfaces served as a proof of principle that STS was able to detect a Kondo resonance at $E_{F}$ $[106,107]$. It became apparent that the line shape (see Figure 3.10a), was not always a peak, but rather a spectral feature of more complex shape as 
we will discuss later*.

The local environment of the impurity has a very strong impact on the shape of the Kondo resonance and temperature. For example, the change from 3 to 4 neighboring atoms, for a Co atom adsorbed on $\mathrm{Cu}(111)$ or $\mathrm{Cu}(100)$ surface changes $T_{K}$ by almost $40 \%$ [108].
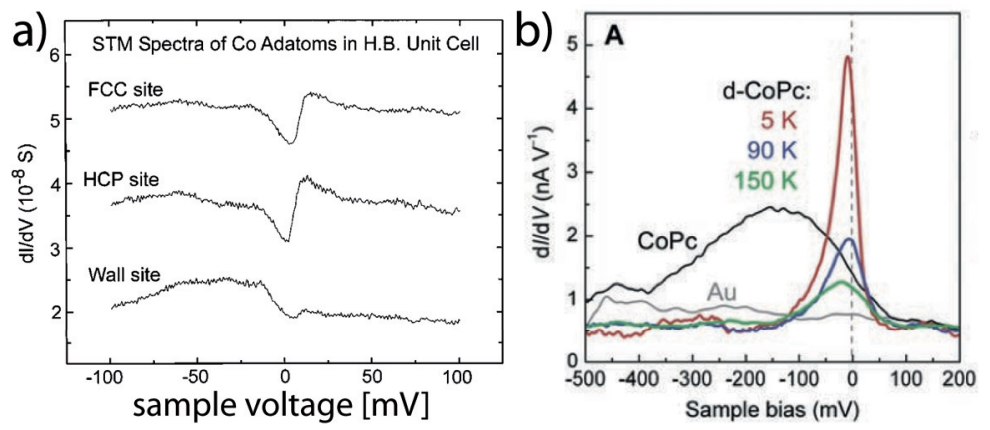

Figure 3.10.: a) STS acquired for a single Co adatom positioned on different sites within the $\mathrm{Au}(111)$ herringbone unit cell. The Kondo resonance remains the same for fcc and hcp sites, but the Fano line shape undergoes a sharp change when the adatom is positioned on a dislocation wall [109]. b) STS spectra taken at the center of a CoPc molecule at $5 \mathrm{~K}$, adsorbed on $\mathrm{Au}(111)$ showing the appearance of a Kondo resonance for deprotonized d-CoPc molecule at 5, 90, and $150 \mathrm{~K}$ (colored lines). Spectra from bare $\mathrm{Au}(111)$ (gray line) is shown for comparison. Image adopted from [110].

The influence of a change in coupling between the magnetic atom and the substrate metal has been further explored by modifying its chemical environment. One approach was to place the magnetic ion inside an organic molecule. Zhao and coworkers found that Co phthalocyanine molecules on $\mathrm{Au}(111)$ are coupled in such a way that no Kondo effect can occur, however when the ligand of the molecule is deprotonized the effective Co - substrate distance becomes larger and the Kondo resonance appears, as shown in Figure 3.10b) [110]. Also within the molecular configuration the local density of states of the substrate plays an important role, e.g. for Fe phthalocyanine molecules on $\mathrm{Au}(111)$ an adsorption site specific Fano line shape and Kondo temperature have been observed [111].

\footnotetext{
"Note that not all features at $E_{F}$ are related to the Kondo effect. However the characteristic increase in width of the resonance peak with temperature, as well as its intensity evolution can be used as proof that a Kondo system is measured [88, 89], see also Eqs. 3.21 and 3.22.
} 
In a more systematic approach the coupling of the impurity spin to the substrates conduction electrons was increased by bonding a different number of CO ligands to a Co atom and hereby increasing $T_{K}$ [112]. A recent study on $\mathrm{CoCu}_{\mathrm{N}}$ clusters showed that the addition of adatoms affects the system in a more complex way than a simple decoupling and that the local and anisotropic electronic structure has to be taken into account [113].

The purposeful manipulation of the density of states at the Fermi level has also been shown to have an influence on the Kondo temperature. The Kondo resonance of $\mathrm{Mn}$-phthalocyanine molecules on $\mathrm{Pb}$ islands grown on $\mathrm{Si}(111)$ manifested oscillating Kondo temperatures as a function of the $\mathrm{Pb}$ film thickness. The crossing of quantum-well states led to oscillations in the density of states at $E_{F}$ [114]. A similar result has been obtained for $\mathrm{Co}$ atoms on $\mathrm{Cu} / \mathrm{Co} / \mathrm{Cu}(100)$ multilayers [115].

Using the manipulation capabilities of an STM setup the exchange interaction of two magnetic atoms in relation to their distance can be investigated using the Kondo effect as a local probe. For Co atoms on $\mathrm{Cu}(100)$ [116], the coupling was found to change with the distance between the Co atoms from ferromagnetic to antiferromagnetic for increasing distances.

\section{The Fano line shape}

In section 2.2 on page 19 we have seen that using an STM it is possible to record a signal that is proportional to the DOS of the sample. In theory the sharp Kondo feature at $E_{F}$ should also be accessible by STS if the system is measured at temperatures below $T_{K}$. Indeed in many STS experiments of Kondo systems a strong feature at $E_{F}$ has been observed. However, the line shape of this feature varies considerably and is not generally a peak, but rather a Fano function. This function was first used to describe the interference of a discrete state with a continuum [117]. In STS measurements of the Kondo resonance the sharp impurity state, giving rise to the Kondo interaction, is intermixing with the continuum of the conduction bands [118].

The exact expression of the Fano function is given by [117]:

$$
\frac{d I}{d V}(\omega)=a \cdot \frac{(q+\epsilon(\omega))^{2}}{1+\epsilon(\omega)^{2}}+b+c \cdot \omega \quad \text { with } \epsilon=\frac{\omega-\epsilon_{k}}{\Gamma}
$$

where $a, b, c$ are fitting constants, and $\omega$ is the energy. The factor $q$ 


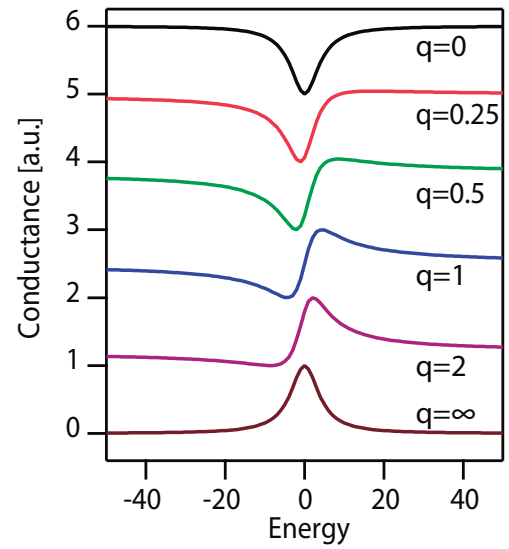

Figure 3.11.: Set of curves calculated with the Fano equation Equation 3.23 for different values of $\mathrm{q}$. At $\mathrm{q}=0$ a Lorentzian dip is detected which corresponds to mainly direct tunneling. In the limit of a large q, a Lorentzian peak is formed corresponding to mainly indirect tunneling. Intermediate values of $\mathrm{q}$ result in an S-like curve. All curves are shifted and normalized for clarity.

determines the line shape, which can be a dip for $q=0$, an asymmetric feature $(q=1)$ or a peak $(q>2)$. For $q=\infty$ the Fano feature becomes a Lorentzian (See Figure 3.11). $\Gamma$ is the half width of the peak which is related to the Kondo temperature by $\Gamma \approx k_{B} T_{K}$. We can therefore use this function to fit the experimental $d I / d V$ peaks, to find a characteristic Kondo temperature. One has to bear in mind the additional broadening introduced by the temperature of the system.

For the interpretation of the $\mathrm{q}$ values found in experiments several models have been proposed. The first model is based on the coherent superposition of two different tunneling channels: a direct coupling channel to the impurity level, and an indirect channel probing the Kondo resonance via the substrate states. The picture implies that the line shape depends on the position of the tip as the contribution of the different tunneling channels would change. A different view states that in STS measurements the tip interacts much more with the substrate than with with the very localized adsorbate states. Conductance measurements probe the presence of a magnetic atom through modified electronic properties of the metal surface. The Fano line shape is therefore thought of as arising from the interference of the Kondo resonance with the continuum of the metal states $[119,120]$. 